\title{
Set-Size Effects for Identification Versus Localization Depend on the Visual Search Task
}

\author{
Tom Busey \\ Indiana University, Bloomington
}

\author{
John Palmer \\ University of Washington
}

\begin{abstract}
In both behavior and neuroscience research, it is debated whether the processing of identity and location is closely bound throughout processing. One aspect of this debate is the possibly privileged processing of identity or location. For example, processing identity may have unlimited capacity, while processing location does not. The authors have investigated the possibility of such privileged processing by measuring set-size effects for a variety of identification and localization tasks. In particular, set-size effects in accuracy visual search are measured with either 1 or 2 possible targets. For 1-target tasks, set-size effects are smaller for identification than localization; for 2-target tasks, set-size effects are larger for identification than localization. The observed crossover interaction is inconsistent with a privileged processing hypothesis for either identity or location. Furthermore, this interaction is predicted by an independent channel model based on signal detection theory, in which the details of each decision determine the relative magnitude of the set-size effects. This result is consistent with the similar processing of identity and location, and it refutes the privileged processing hypothesis for either identity or location.
\end{abstract}

Keywords: attention, visual search, binding, spatial localization, signal detection theory
Several lines of research have suggested that "what" and "where" information may be treated differently by the visual system. Anatomical and physiological research has suggested that the ventral pathway specialized for identification and the dorsal pathway specialized for localization (Haxby et al., 1994; Ungerleider \& Mishkin, 1982; van Essen \& Gallant, 1994). In the context of visual search, the possibility of distinct pathways has motivated proposals for differential effects of divided attention on the processing of identity and location. In one proposal, Treisman and Gelade (1980; for a review, see Quinlan, 2003) have suggested unlimited-capacity processing of identity information but not location information. Thus identity has a privileged role. In an opposing proposal, Sagi and Julesz (1985a, 1985b; Donk \& Meinecke, 2001) have suggested unlimited-capacity processing of location but not identity. Here location has a privileged role. In this article, we test predictions of such privileged processing hypotheses as well as the alternative of similar processing of identity and location information (e.g., Baldassi \& Verghese, 2001; Johnston \& Pashler, 1990). In particular, we capitalize on analyses of the magnitude of set-size effects in psychophysics to make a comparison of set-size effects in identification and localization tasks (e.g.,

Tom Busey, Department of Psychology, Indiana University, Bloomington; John Palmer, Department of Psychology, University of Washington.

We thank Jim Johnston, Steve Luck, Jeff Miller, and Jeremy Wolfe for criticism and suggestions on drafts of the article. We also thank Bill Prinzmetal and Mieke Donk for their assistance in the review process.

Correspondence concerning this article should be addressed to Tom Busey, Department of Psychology, Indiana University, Bloomington, IN 47405; or to John Palmer, Department of Psychology, Box 351525, University of Washington, Seattle WA 98195-1525. E-mail: busey@indiana.edu or jpalmer@u.washington.edu
Palmer, 1995). This comparison highlights the complexity of casual task comparisons and thus the need for careful task analyses and modeling before one accepts claims of privileged processing for either localization or identity.

\section{Theoretical Background}

Below, we summarize three hypotheses in the extant literature that bear on the relation between identification and localization and that make predictions for set-size effects in visual search and related tasks.

\section{Three Hypotheses}

Identification without localization. By this hypothesis, identification is possible without localization in an early stage of processing. For example, in feature integration theory (Treisman \& Gelade, 1980; Treisman \& Gormican, 1988; for a review, see Quinlan, 2003), there is a parallel, unlimited-capacity stage that processes simple feature information followed by a serial stage necessary for processing relations among features. It is further assumed that discrimination of simple features is mediated by a pooled response from the first stage. This pooled response loses location information but preserves identity. Localization requires processing by a further serial stage. Thus set-size effects are predicted to be larger for localization than identification (response time: Saarinen, 1996; accuracy: Bennett \& Jaye, 1995). Variations of this hypothesis can be found in feature and item perturbations (Lee \& Estes, 1981; Wolford, 1975; Wolford \& Shum, 1980) and in the differential loss of identity and location information in very-short-term visual memory (Irwin \& Brown, 1987; Mewhort, Campbell, Marchetti, \& Campbell, 1981; Mewhort, Huntley, \& Duff-Fraser, 1993). 
Localization without identification. By this hypothesis, localization is possible without identification in an early stage of processing. Sagi and Julesz (1985b; see also Donk \& Meinecke, 2001; Müller \& Rabbitt, 1989) have proposed a parallel, unlimited-capacity stage that allows detection and localization of targets followed by a serial stage that is necessary to identify targets. The first stage localizes targets by detecting feature gradients that are made by an "odd" stimulus. Identification requires a second serial stage. Thus, set-size effects are predicted to be larger for identification than localization (accuracy search: Sagi \& Julesz, 1985b). Such a feature contrast hypothesis has been investigated in detail by Nothdurft $(1985,1991,1992,1993)$. He has demonstrated a variety of conditions where visual search depends on the local feature contrast rather than the specific values of the features.

Similar processing of identity and location. By this hypothesis, similar processes mediate identification and localization. For example, Johnston and Pashler (1990; see also Atkinson \& Braddick, 1989; Bloem \& van der Heijden, 1995) have suggested that a two-stage search theory (Hoffman, 1979; Treisman \& Gelade, 1980) be modified so that a detection by the first stage calls attention to the relevant information for that stimulus, which includes both identity and location information. Thus, set-size effects are predicted to be similar for identification and localization, all else equal (accuracy search: M. Green, 1992). This hypothesis is implicit in signal detection theories of identification and localization (e.g., Baldassi \& Verghese, 2001; Swensson \& Judy, 1981). Such similar processing can be implemented by either close binding and common processing or by similar processing of separate representations. Thus, while support for privileged processing is consistent with separate representations of identity and location information, evidence against privileged processing does not allow one to reject separate representations of identity and location information. Rather, it removes one line of support for the hypothesis.

\section{Johnston and Pashler's Critique}

Most of the initial tests of these hypotheses depended on a theoretic comparisons between identification and localization performance. While these comparisons have motivated this research, their interpretation depends on three problems nicely described by Johnston and Pashler (1990).

Different properties problem. Some tasks offer more information for one kind of judgment relative to another. Consider an extreme example: Suppose one is to judge orientation of a line stimulus as either leaning left or right of vertical. In addition, one must judge if the line was to the right or left of fixation. Only a single line is presented at one or the other side of fixation. The problem arises because different information is sufficient to make the two judgments. The orientation of the line is necessary for the orientation judgment, but any detectable attribute is sufficient for the location judgment. To make the tasks comparable, the target must be distinguished from the distractors only by an attribute sufficient for identification. For example, one can place lines on both sides of fixation that differ only in orientation.

Negative information problem. The next issue is whether guessing can differentially affect identification and localization. Suppose one is performing a disjunctive search with a color target and a shape target (e.g., Treisman \& Gelade, 1980). Further, suppose the color target is easier to detect than the shape target. Then, on trials in which there is no obvious color target, one can improve performance by guessing a shape target and never guessing a color target. In this situation, guessing can improve identification without improving localization. Here, this problem is addressed by using explicit decision models.

Location-reporting problem. The three hypotheses refer to the internal representation of location, not the reported location. Errors may arise in translating the internal representation to a response. Indeed, if the stimulus locations are close together and confusable, such mislocalizations are inevitable. Here, we consider explicit decision models in combination with widely separated locations and an analysis of mislocalization errors.

These issues have been addressed in several ways. Swensson and Judy (1981) applied signal detection theory to visual search tasks that required either identification or localization. Johnston and Pashler (1990) examined the correlation between identification and localization accuracy on a single trial. Marc Green (1992) used the two-by-two paradigm to compare identification and localization with carefully matched tasks. Each of these methods have strengths and weaknesses. In this article, we further pursue the relative magnitude of set-size effects in accuracy search to test the privileged processing hypotheses.

\section{Set-Size Effects in Visual Search}

By the privileged processing hypothesis, unlimited-capacity processing is possible for identity or location but not both. To address this possibility, one must determine how to measure setsize effects across a variety of tasks and stimuli. For example, how does one compare the accuracy in a yes-no task with the accuracy in an eight-alternative localization response? Another key issue is the interaction between the difficulty of discrimination and set size: More difficult discriminations result in larger effects of set size on accuracy and time. We describe two approaches to characterizing set-size effects that are both based on signal detection theory.

\section{Two Approaches to Measuring Set-Size Effects}

The $d^{\prime}$ approach. Swensson and Judy (1981) were one of the first to compare set-size effects for identification and localization. Observers viewed simulated medical images with pixel noise. Images contained a large ring structure with a superimposed small disk target. The target could appear in one to eight pre-specified locations. Performance was measured in several tasks including yes-no identification and $n$-alternative localization. For each task, an appropriate $d^{\prime}$ measure was estimated for each set size. They developed the ideas of Tanner (1961) and others in predicting that uncertainty decreases performance even if the representation of the multiple stimuli are independent. By using the tailored $d^{\prime}$ measure, they showed that the set-size effects were similar for identification and localization. Swensson and Judy concluded that a simple model based on signal detection theory and a common perceptual representation can account for both identification and localization performance. Examples of similar analyses include Burgess and Ghandeharian (1984); Cameron, Tai, Eckstein, and Carrasco (2004); Creelman and Macmillan (1979); and Eckstein and Whiting (1996). 
The threshold approach. An alternative approach was described by Palmer, Ames, and Lindsey (1993). First, rather than compare performance by using a $d^{\prime}$ measure tailored for each task, they compared tasks by using a threshold measure that was defined by a particular proportion correct. This measure makes differences between the tasks explicit, and they can be compared with the differences predicted by any theory. Consider next the thresholdversus-set-size function. This function describes the set of stimuli and set sizes that yield equivalent performance. Such equivalences allow tests of general theories of performance (e.g., Loftus, Oberg, \& Dillon, 2004). To reduce the magnitude of the set-size effect to a single value, one can use a threshold ratio because the underlying model is essentially multiplicative, and a threshold ratio removes the unit of the stimulus. Furthermore, one can normalize the threshold ratio by the ratio of set sizes. In particular, Palmer et al. (1993) borrowed a measure from studies of light adaptation. The $\log$-log slope is the log of the threshold ratio relative to the log of the set-size ratio. This measure allows one to make rough comparisons across different combinations of set size. The advantage of this measure is that, because it is a ratio measure, it is unitless and does not depend on the sensitivity or overall ability of any given participant. By using the log-log slope measure, they derived predictions for several search tasks. For yes-no search tasks, set sizes of two to eight are predicted to have a log-log slope of 0.22 . For $n$-alternative localization, the same set sizes are predicted to have a log-log slope of 0.34 (Shaw, 1980; see our Appendix for details). Thus, by this measure, identification is predicted to have smaller set-size effects than those of localization.

The $d^{\prime}$ and threshold approaches are based on a common theory. For fitting a single data set to a single theory, they are essentially equivalent. Differences between the approaches become evident when considering the predictions relative to other theories. The advantage of the threshold approach is to facilitate explicit evaluation of alternative theories by using the descriptive threshold measure rather than building the theory into the measure of performance. In summary, we follow the threshold approach both to make explicit the task differences and to address the interaction between discriminability and set size. In the next two sections, we review the set-size effects found for identification and localization for two kinds of search tasks: Those with one possible target and those with two possible targets. This review reveals that set-size effects are dependent on this aspect of the task.

\section{One-Target Studies}

Perhaps the simplest example of identification is a yes-no task. Several studies have examined a variety of attributes by using the threshold approach to compare across discriminability (contrast: Palmer, 1994, and Foley \& Schwarz, 1998; color: Monnier \& Nagy, 2001, and Palmer, 1994; size: Palmer et al., 1993, and Palmer, 1994; orientation: Palmer, 1994; motion speed: Palmer, Verghese, \& Pavel, 2000; motion direction: Dobkins \& Bosworth, 2001; shape: Palmer et al., 1993). For set sizes 2 and 8, the $\log -\log$ slope for all of these attributes was between 0.2 and 0.3 , which compares well with the 0.22 predicted by a noisy integration model with unlimited capacity (Palmer et al., 1993; Shaw, 1980).

There are several studies that have directly compared identification and localization by using the $d^{\prime}$ approach (e.g., Swensson \& Judy, 1981). Other studies have closely examined set-size effects for localization of simple attributes (e.g., Shaw, 1980). Here, we extend those studies by using the threshold approach to compare identification and localization. Given the indirect evidence, we fully expect that for one target the set-size effects for identification will be smaller than for localization.

\section{Two-Target Studies}

Quite different results for the identification-localization comparison have been obtained for orientation accuracy search with two possible targets (only one of which was present on any given trial). In Baldassi and Burr (2000), the targets were either right leaning or left leaning with vertical distractors. Observers made either a choice between left and right leaning (two-target identification) or a choice among $n$-alternative locations. By using the threshold approach, they found set-size effects for identification were larger than set-size effects for localization. Specifically, the $\log -\log$ slope was 0.5 for identification and was about 0.2 for localization. This is the opposite pattern observed for one-target search. Baldassi and Verghese (2001) developed a noisy integration model specific to the two-target task. They showed that a model with similar processing of identification and localization predicts this ordering of set-size effects for two-target identification and localization. This result has been replicated and extended by Solomon and Morgan (2001).

\section{Summary of Prior Research}

In summary, Swensson and Judy (1981), Baldassi and Verghese (2001), and others have argued that models based on signal detection theory can account for differences in set-size effects between identification and localization. Unnoticed was that the one-target and two-target studies produced the opposite pattern of set-size effects for identification and localization. Such a reversal is not consistent with the privileged processing hypotheses suggested by either Treisman and Gelade (1980) or by Sagi and Julesz (1985b). We pursue this observation with a direct comparison of identification and localization for one- and two-target search tasks. We also include in the Appendix an analysis of the different decision problems imposed by each task.

\section{General Method}

Figure 1 shows an example display. Observers fixate a central cross, and either two or eight grating patches appear around a fixation cross. The target in this example is a grating patch tilted to the left, and depending on the task, the observer identifies the target or reports its location. We tilt the individual gratings to vary the angle between the target and distractors. If both angles are close to vertical, this is a difficult task. However, as both stimuli are tilted away from vertical in opposite directions, this task becomes trivially easy. Systematically varying the angular difference between targets and distractors allows the measurement of a psychometric function and the estimation of an angle difference threshold. How much this threshold increases with additional stimuli is our measure of the set-size effect.

One difficulty that arises during comparisons of identification and localization is that the details of the task matter. As a result, we considered a variety of visual search tasks, along with a model of 


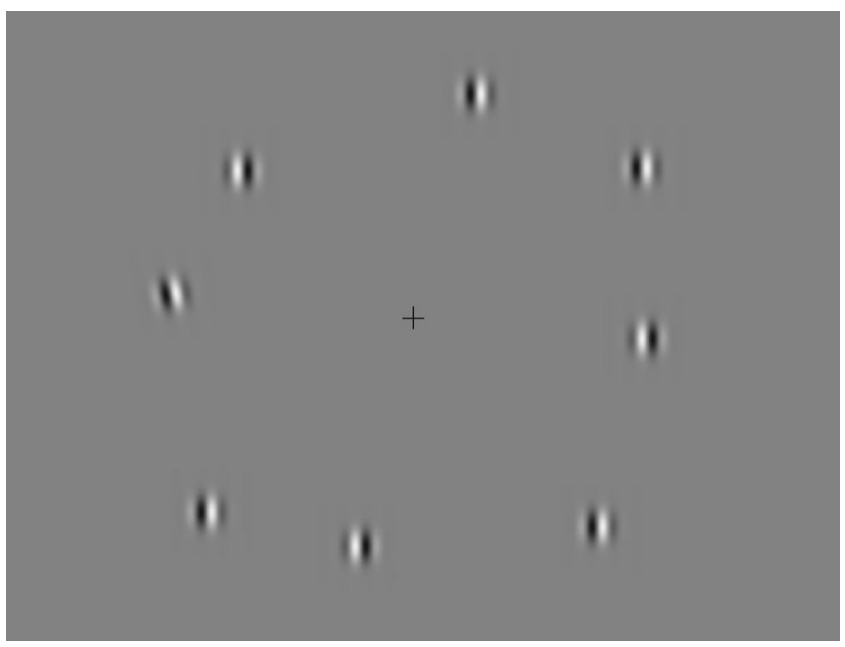

Figure 1. Example display with set size 8 as used in Experiment 1.

each task that embodies the structure of the decision necessary for that particular task. The most common task involving identifying a target item is a yes-no judgment of the presence of the target (yes-no identification). The most common localization task differs in that the target is always present and the observer indicates its location (n-alternative localization). We also employed a variation on localization in which the observer indicates if the target was on the left side or right side of the display (coarse localization). These tasks were also investigated with two targets, in which only one tilted target was present on each trial, but it could be one of two tilts (left or right leaning). For identification, one of two targets is always presented among distractors, and the observer indicates the identity of the target (two-target identification). For localization, the observer reports the location of the single target (of two possible) in terms of the $n$ possible locations (two-target, $\mathrm{n}$-alternative localization) or in terms of the left or right side of the display (two-target, coarse localization).

\section{Observers}

The observers were young adults with normal or corrected-tonormal vision who received monetary compensation.

\section{Stimuli}

An example display is found in Figure 1. The stimuli were 1.5 cycles per degree (cpd) cosine gratings presented in a Gaussian window with a full width of $1^{\circ}$ at half height. For most experiments, targets were tilted and distractors were vertical. The stimuli were arranged around an iso-eccentric circle about $7^{\circ}$ in the periphery. These locations were modified in two ways. First, each stimulus was jittered by up to $0.8^{\circ}$ to avoid accidental alignments among the stimuli. Second, for experiments involving coarse localization as one of the tasks, the stimuli were shifted horizontally to make it unambiguous which were on the left and right sides of the displays. The phase of the grating was also randomized across stimuli.

The gratings were displayed on a 21-inch Macintosh grayscale monitor with luminance determined by a Video Attenuator and the
VideoToolbox software library (Pelli, 1997). The surround luminance was $40 \mathrm{~cd} / \mathrm{m}^{2}$. The contrast of the gratings was determined in different ways depending on the experiment. It was always a value several times the contrast threshold for these peripheral stimuli. For some experiments, a single fixed level of contrast was used for all observers and conditions. For other experiments, a preliminary measurement was conducted for each observer in which contrast sensitivity was determined for a single grating displayed in the periphery to the left or right of fixation. The contrast used for the search experiment was some multiple (e.g., 3) of the measured threshold contrast. The same value of contrast was always used for corresponding identification and localization tasks.

\section{Procedure}

The display contained a fixation cross that never disappeared, and observers were instructed to maintain fixation during a trial. Each trial began with a warning tone, which was followed by the onset of the stimulus after $30 \mathrm{~ms}$. Depending on the experiment, the stimuli were either presented abruptly with a very short duration (e.g., $100 \mathrm{~ms}$ ) or were presented with a gradually changing Gaussian time course (e.g., effective duration $\sim 200 \mathrm{~ms}$ ). After the stimulus offset, the observer made an unspeeded response appropriate for the task. For yes-no identification, the observer pressed the 8 key if the target was present and the 9 key if all items were distractors. For two-target identification, the observer pressed the 5 key for a left-leaning target and the 6 key for a right-leaning target. For $n$-alternative localization, the eight keys around the 5 key on a numeric keypad were designated to correspond to the eight possible stimulus locations. For coarse localization, the observer pressed the 2 key for the target on the left side and the 3 key for the target on the right side. After response, feedback was given with high and low tones indicating correct and incorrect, respectively. The next trial occurred after an additional $500 \mathrm{~ms}$.

For all tasks, performance was measured at set size 2 and set size 8 . For set size 2 , the two stimuli were always opposite each other to further minimize possible eye movements. A block of 384 trials served as practice for each observer and allowed the experimenter to watch for eye movements by the observers and provide verbal feedback if they made eye movements. This practice session also established an initial angle difference threshold for each condition by using the QUEST procedure (Watson \& Pelli, 1983). For the two-choice tasks, the procedure varied the difference in the angle between the target and the distractors so that on average the observer was $75 \%$ correct, which is halfway between chance and perfect. For the $n$-alternative localization task, this was modified because chance was $12.5 \%$ in the $n$-alternative localization task for set size 8 . For this case, the angle was varied to achieve $56.25 \%$ correct, which is halfway between chance and perfect. The actual experiment measured performance at six logarithmically spaced angles centered around the initial threshold. Each angle was tested in a separate block in order to allow observers to adjust their yes-no criterion to maintain an equal bias. Blocking also allows an estimate of the hit and false alarms for each angle separately. At the end of each block of trials, observers were cautioned if their responses in the yes-no task exhibited a bias. Specifically, they were warned if their percentage of one response type exceeded $60 \%$ and were asked to respond more equally. Observers com- 
pleted one task and then the other, but in different orders. Performance in the yes-no task was averaged across the target present and target absent trials, under the assumption of equal bias.

\section{Analysis of Psychometric Functions}

The most theoretical approach to the analysis of psychometric functions is to choose a function for a single stimulus discrimination (such as the cumulative Gaussian) and then derive how the function changes with set size (Palmer et al., 2000; Pelli, 1987). These models predict that increasing set size steepens the slope of the psychometric function as well as increasing the threshold. We focus on estimating the threshold because threshold estimates are more reliable than estimates of the slope. Accordingly, we fit a standard psychometric function with two parameters. The two common models in the literature are the Weibull (D. M. Green \& Luce, 1975; Quick, 1974) based on high threshold theory and the generalized Gaussian based on signal detection theory (Nachmias \& Kocher, 1970; Pelli, 1987). Both models have a threshold and slope parameter and make virtually identical estimates of the thresholds for the experiments reported here. We report the Gaussian results only because the Gaussian is more compatible with signal detection theory. In sum, we focus on predictions of the effect of set size on thresholds rather than the detailed shape of the psychometric functions.

\section{Experiment 1}

\section{Method}

In Experiment 1, set-size effects on the angle difference threshold were measured for four tasks. Two were one-target tasks: yes-no identification and coarse localization; and two were twotarget tasks: two-target identification and two-target, coarse localization. Targets were tilted and distractors were vertical for all four tasks. The contrast of the stimuli was defined as $\mathrm{C}=(\mathrm{L}-\mathrm{S}) / \mathrm{S}$, where $\mathrm{L}$ is the maximum luminance of the grating and $\mathrm{S}$ is the mean surround luminance. Contrast was fixed throughout at $48 \%$, which is the largest contrast without clipping for these stimuli and several times the contrast threshold in the periphery. After practice, twelve 1-hour-long sessions resulted in 864 trials per psychometric function. Six observers participated in this experiment.

\section{Results}

The results for one observer, MB, are illustrated in Figure 2. The panels on the left side of the figure show the results for the tasks with one target. Begin by considering Panel A. The psychometric functions are shown for yes-no identification with one target. They plot the proportion correct as a function of the difference in angle between the target and distractors. The filled circles indicate the proportion correct for set size 2 , and the filled triangles indicate the proportion correct for set size 8 . There is a clear effect of set size for the entire range of angles. The points are fit with a cumulative Gaussian raised to a power as described in the general methods (Pelli, 1987). The dotted grid line marks the proportion correct of 0.75 , which is halfway between chance and perfect, and the dotted drop lines to the $\mathrm{x}$-axis show the estimated difference thresholds defined by this performance level. For a good first approximation,
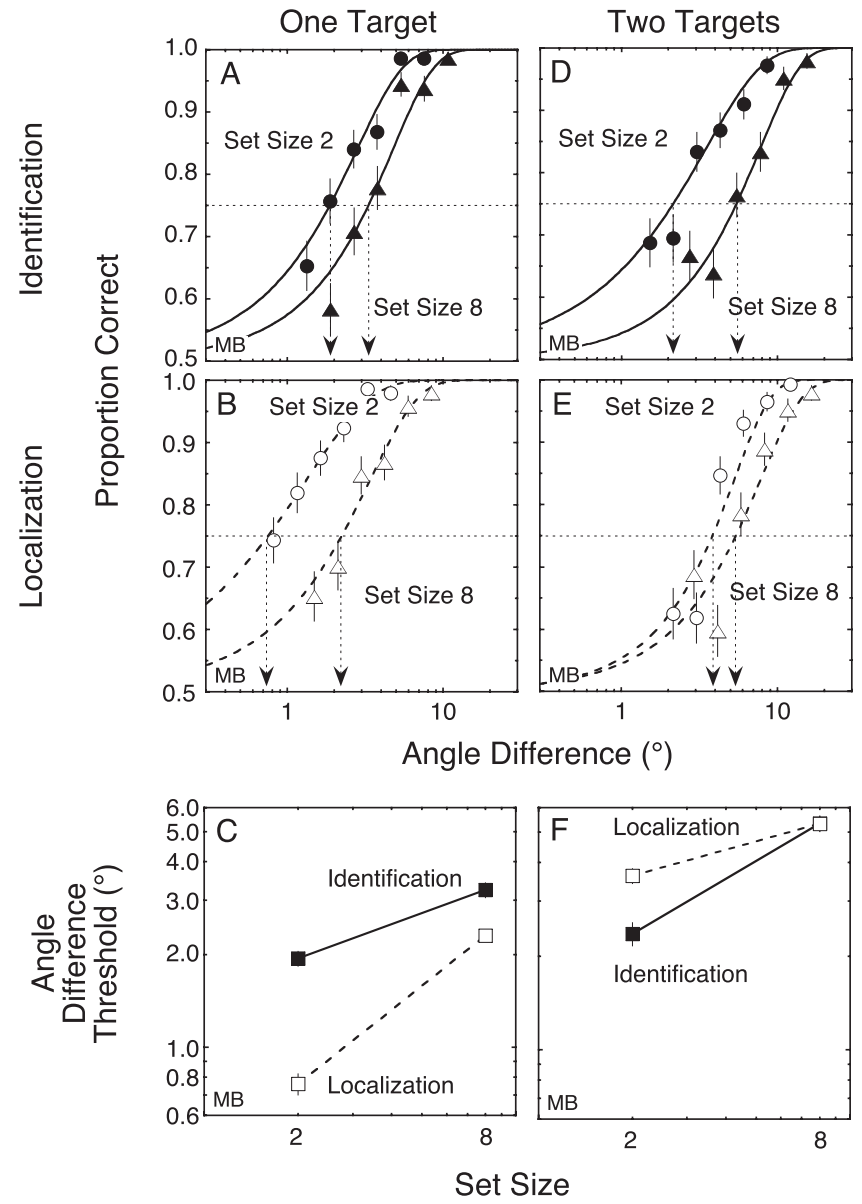

Figure 2. The results of Experiment 1 are illustrated for one observer, MB. The left column of panels shows the results with one target; the right column of panels shows the results for two targets. The top row of panels is for identification tasks; the middle row is for localization tasks. For each of the four top panels, the proportion correct is plotted as a function of the angle difference. The effect of set size can be summarized by the horizontal shift of the two functions within each panel. This horizontal shift is larger for localization with one target and larger for identification for two targets. The bottom two panels summarize this interaction by plotting the angle difference threshold as a function of set size. Error bars represent one standard error. Identification is represented with solid lines and localization with dashed lines. In Proportion Correct panels, horizontal lines reflect the threshold performance level (typically midway between chance and perfect performance), and vertical lines represent the estimated threshold in units of degrees of angle difference.

the set-size effects can be summarized as a horizontal shift of these psychometric functions.

In the Panel $\mathrm{B}$, the corresponding psychometric functions are shown for coarse localization with one target. Here the open symbols and dashed curves illustrate the psychometric function. In terms of proportion correct, the set-size effect is clearly larger for localization than it is for identification. This can be quantified in terms of the threshold for each set size. As above, the dotted lines show the estimated difference thresholds. The change in threshold is clearly larger for localization than for identification.

In the Panel $\mathrm{C}$, the angle difference thresholds are summarized as a function of set size for both tasks. Thresholds are higher for 
identification than for localization. However, the effect of set size is smaller for identification than for localization. Specifically, the $\log -\log$ slope is 0.37 for identification and 0.81 for localization.

The panels on the right side of Figure 2 show the corresponding results for tasks with two targets. In Panel D, the set-size effect is shown for two-target identification. It is clearly larger than found for one-target identification. In Panel E, the set-size effect is shown for two-target localization. It is clearly smaller than found for one-target localization. This reversal in the magnitude of effects is summarized by the threshold-versus-set-size graph in Panel F. The $\log -\log$ slope is 0.59 for two-target identification and is 0.28 for two-target localization. This pattern of results is repeated for all 6 observers.

The mean thresholds for the 6 observers are illustrated in Figure 3. Panels A and B show threshold-versus-set-size functions for one versus two targets, respectively. As with the observer $\mathrm{MB}$, there is a reversal in the magnitude of set-size effects. For one-target tasks, the log-log slope of $0.40 \pm 0.05$ for identification is smaller than the $\log -\log$ slope of $0.75 \pm$ 0.08 for localization. For two-target tasks, the log-log slope of $0.60 \pm 0.04$ for identification is larger than the $\log -\log$ slope of $0.32 \pm 0.06$ for localization. This comparison is made explicit in Panel $\mathrm{C}$ of Figure 3. It plots the log-log slope for one- and two-target conditions. The solid symbols are for identification, and the open symbols are for localization. For this measure of

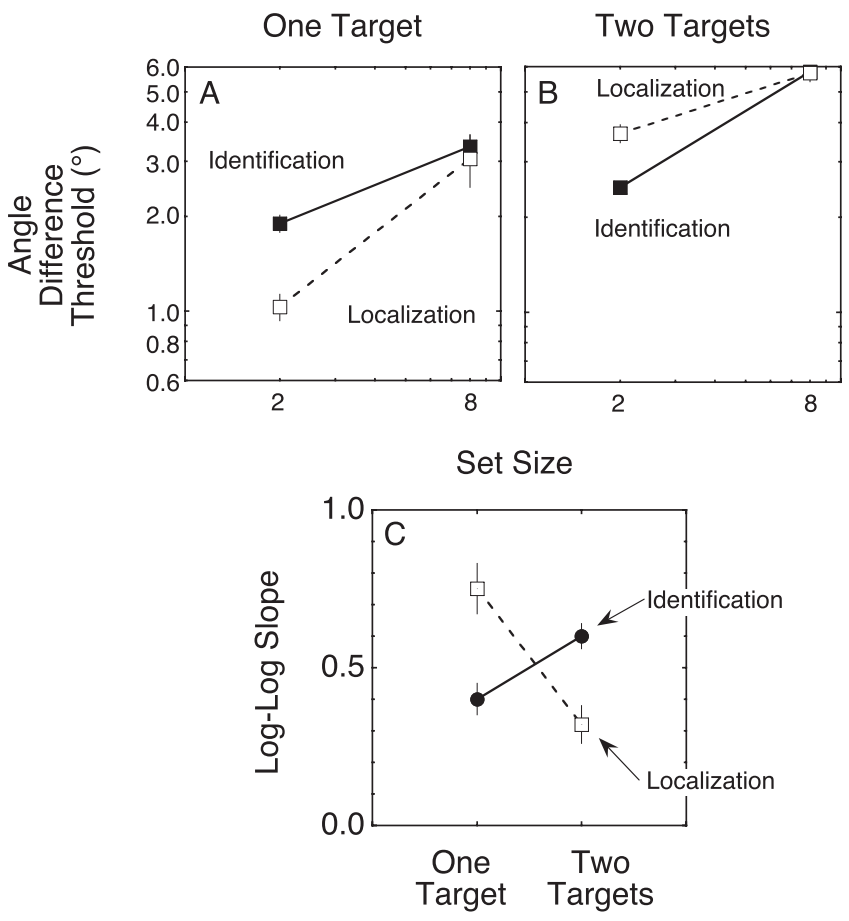

Figure 3. The results of Experiment 1 are summarized by the mean of estimates from all 6 observers. The top two panels show the threshold as a function of set size. For the mean threshold, the set-size effects are larger for localization for one target and larger for identification for two targets. The bottom panel summarizes this crossover interaction by plotting the $\log -\log$ slope as a function of the number of targets. Error bars represent one standard error. Identification is represented with solid lines and localization with dashed lines. set-size effects, there is a crossover interaction between the task and the number of targets. The interaction measure for the effect of task with one target minus the effect of task for two targets yields a value of $0.64 \pm 0.07, t(5)=8.8, p<.001$. Moreover, this crossover interaction is found individually for all 6 of the 6 observers. Thus, this interaction is reliable and uniform across observers.

\section{Discussion}

The privileged processing hypotheses predict that set-size effects are critically dependent on whether the task is identification or localization. The results of Experiment 1 show that this is not the case. The relative magnitude of set-size effects for identification and localization is reversed when one compares one-target and two-target tasks. Thus, on the face of this result, one must reject the privileged processing hypotheses.

One can question the assumptions of the threshold analysis rather than the privileged processing hypothesis. Indeed, the use of thresholds as a summary of performance is imperfect. In particular, it is only a complete summary of performance if the shape of the psychometric functions is consistent over all of the conditions of interest. The slope of these functions do vary with set size (e.g., Palmer et al., 2000). This effect is not large, but it introduces a complication. How can one compare set-size effects more generally?

To make a more general comparison, one can compare the observed proportion correct for an appropriately chosen condition. The trick is to find a way to match conditions across different tasks. The threshold analysis does this by estimating thresholds for all set sizes. This uses a common performance level to compare stimulus conditions. Alternatively, one can estimate a single critical stimulus value for a particular set size to define a point of comparison across tasks. For example, find an angle that results in $75 \%$ correct for set size 8 . Then for that angle, estimate the proportion correct for the set-size- 2 condition. The better the performance in set size 2, the larger the set-size effect. This approach is illustrated in Panel A of Figure 4. It is a schematic of proportion correct as a function of angle for two set sizes. It is similar to the panels shown in Figure 2 in the analysis of Experiment 1 . The critical angle is defined by $75 \%$ performance in the set-size- 8 condition. This rule allows one to find stimuli with equivalent difficulty across tasks. Then, the proportion correct at this critical angle can be estimated for each task. This estimation uses the psychometric functions but does not assume anything about a consistent slope. Indeed, in the analysis of Experiment 1, each observer was fit with a free parameter for both the $75 \%$ correct threshold and the slope.

The results of this alternative analysis are shown in Panel B of Figure 4. For example, identification in the one-target task yielded an estimated proportion correct of $88 \%$, which is shown by the solid point on the left side of the figure. Similar estimates were made for all four tasks. For one-target tasks, the estimated proportion correct is higher for localization than for identification. Thus, the set-size effect is larger for localization than for identification. This is consistent with the results of Experiment 1 as summarized by thresholds. For the two-target tasks, the proportion correct is higher for identification than for localization. Again, this is consistent with the threshold analysis. Thus, by this method of 

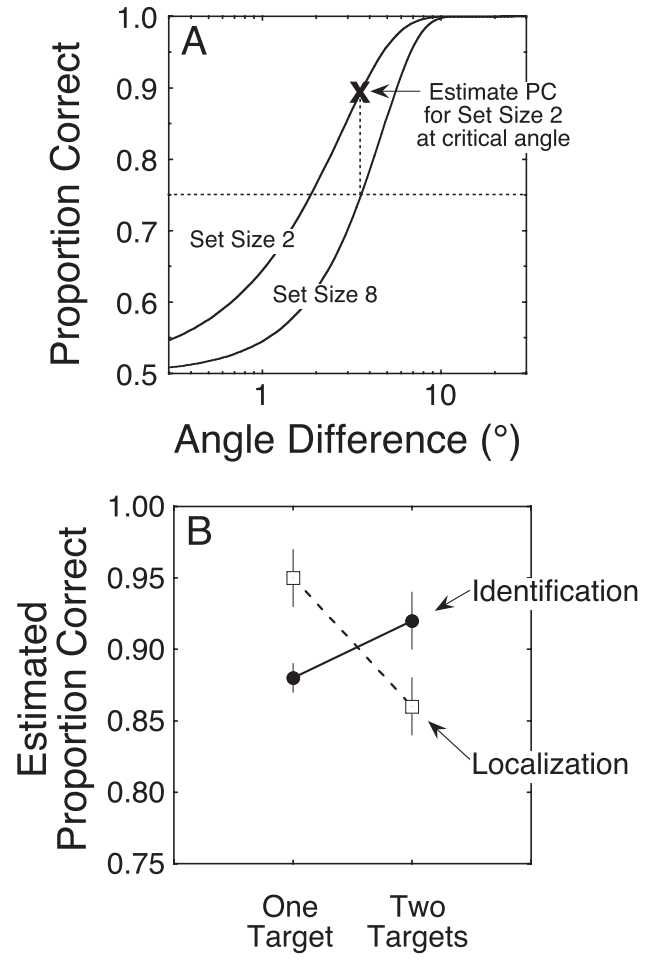

Figure 4. The set-size effects of Experiment 1 are measured in a way that allows for the psychometric functions to vary in steepness. Panel A illustrates how the proportion correct (PC) is estimated for set size 2 from the angle difference that is at threshold for set size 8. For this estimate, larger proportion correct values indicate larger set-size effects. Panel B shows the estimated proportion correct as a function of the task and the number of targets. This more general analysis results in the same crossover interaction as found with the threshold analysis. Error bars represent one standard error. In Panel A, the horizontal dashed line represents our chosen threshold performance criterion $(75 \%$ correct identification or localization), and the vertical dashed line reflects the increase in accuracy for set size 2 relative to set size 8 at the set size 8 threshold angle. In Panel B, identification is represented with solid lines and localization with dashed lines.

estimating the proportion correct, there is also a crossover interaction. The interaction measure for the effect of task with onetarget minus the effect of task for two-targets yields a value of $13 \pm 2 \%, t(5)=5.8, p<.005$. Moreover, this interaction is evident in 6 of the 6 observers and is a crossover interaction in 5 of the 6. In sum, this more general analysis yields essentially identical results as the threshold analysis. By both analyses, one must reject the privileged processing hypotheses.

The complexity of these tasks makes casual comparisons difficult. However, we offer a straightforward generalization of signal detection theory as a unifying model that builds in the differences between the tasks and provides not only a qualitative account of the ordering of the set-size effects but also provides intuitions about the surprising differences between the tasks. First, however, we introduce an $n$-alternative localization task to demonstrate how the results are not dependent on our particular choice of localization task.

\section{Experiment 2}

To generalize the results of Experiment 1, we next consider the same comparison, but for the more common $n$-alternative localization task rather than for coarse localization. The special feature of coarse localization is that it maintains a two-choice response for all set sizes. In contrast, the $n$-alternative localization task varies the number of response alternatives with set size. This dramatically changes the chance level of performance and without some kind of theory, one cannot compare proportion correct measures for different set sizes. In the following experiment, we obtain psychometric functions for all four tasks at two set sizes as done with Experiment 1. To make comparisons, we use a threshold analysis with the threshold defined as halfway between chance and perfect. This approach is motivated by both a high threshold "correction for guessing" and by a signal detection analysis that is described in the Appendix. With the assumptions, we can attempt to generalize the results of Experiment 1 to the more common $n$-alternative localization task.

\section{Method}

Experiment 2 repeats much of the design of Experiment 1. The new feature was to replace the coarse localization with the more common $n$-alternative localization. Other details of the design differ because some refinements were missing due to conducting this experiment before Experiment 1. In particular, it was conducted as two separate studies with different observers in the one-target and two-target conditions. For the one-target conditions, the target was tilted, and distractors were tilted in the opposite direction. For the two-target conditions, the target was tilted one way or the other, and the distractors were vertical. The contrast of the stimuli was three times the measured contrast threshold for each observer. On average, this was $16 \%$ for the one-target tasks and $15 \%$ for the two-target tasks. The stimuli were presented with a Gaussian time course that extended for $280 \mathrm{~ms}$ in total. This was done in part because we thought that the abrupt onsets of the 100-ms duration might introduce transients that might favor one task over another and interact with set size. Because of the long tails of this Gaussian time course, the effective duration was approximately $150 \mathrm{~ms}$. Thus, we argue that there was not enough time for an eye movement before the end of the effective display. This experiment also had fewer trials than Experiment 1. After practice, four 1-hour-long sessions resulted in 576 trials per psychometric function. Six observers participated in the one-target conditions and 5 in the two-target conditions.

\section{Results}

The results for one pair of observers (MR and MD) are illustrated in Figure 5. In Panel A, the psychometric functions are shown for yes-no identification with one target (observer MR). As with Experiment 1, there is a clear effect of set size for the entire range of angles, and the set-size effect can be summarized by a horizontal shift of these functions.

In Panel B, the corresponding psychometric functions are shown for $n$-alternative localization with one target for the same observer. In terms of proportion correct, the set-size effect is clearly larger for localization than it was for identification. Unfortunately, this 

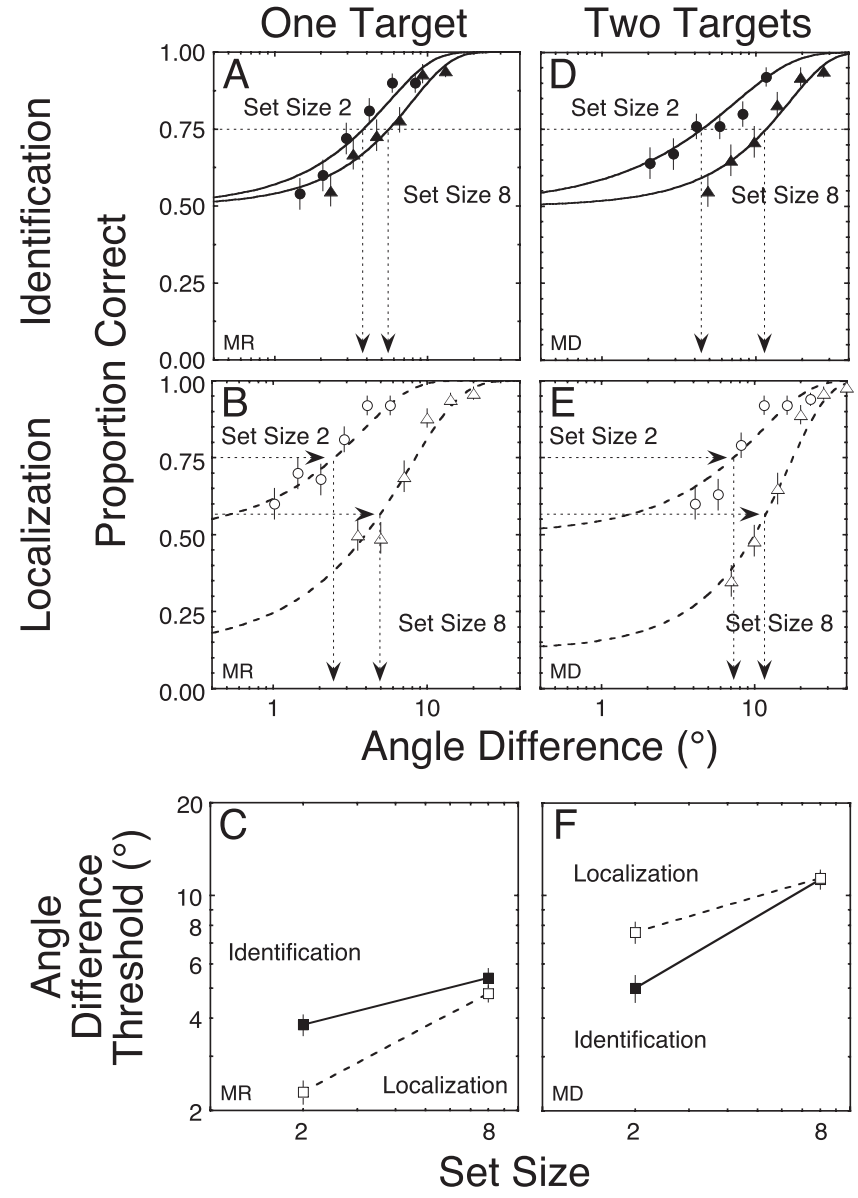

Figure 5. The results of Experiment 2 are illustrated for observer MR with one target and observer MD with two targets. As with Figure 2, the left and right columns show the results for one and two targets, respectively. The top two rows show the results for identification (solid lines) and localization (dashed lines). The effect of set size can be summarized by the horizontal shift of the two functions within each panel but with the additional provision of different chance levels of performance for different set sizes in localization. This horizontal shift is larger for localization with one target and larger for identification with two targets. The bottom two panels summarize this interaction by plotting the angle difference threshold as a function of set size. The threshold is defined by the performance level that is halfway between chance and perfect. Error bars represent one standard error. In Proportion Correct panels, horizontal lines reflect the threshold performance level (typically midway between chance and perfect performance), and vertical lines represent the estimated threshold in units of degrees of angle difference.

comparison is made difficult by the change in the chance level with changes in set size. A better comparison is in terms of the threshold for each set size. This assumes that set size causes both a vertical scaling and a horizontal shift of the psychometric function. As in Experiment 1, the dotted lines show the estimated difference thresholds. The change in threshold is larger for localization than for identification.

In Panel $\mathrm{C}$, the angle difference thresholds are summarized as a function of set size for both tasks for observer MR. Identification thresholds are higher than localization thresholds. However, the effect of set size is smaller for identification than for localization. Specifically, the log-log slope is 0.26 for identification and 0.54 for localization. This qualitatively replicates the results for Experiment 1 .

The panels on the right side of Figure 5 show the corresponding results for the tasks with two targets and observer MD. In Panel D, the set-size effect is shown for two-target identification. It is larger than found for one-target identification. In Panel E, the set-size effect is shown for two-target localization. Again the comparison is made difficult by the changing chance level of performance. By using the threshold illustrated by the dotted lines, the set-size effect is smaller for two-target localization than for two-target identification. This reversal in the magnitude of effects is summarized by the threshold-versus-set-size graph in Panel F. The log-log slope is 0.59 for two-target identification and is 0.29 for two-target localization. This pattern of results is repeated for all 6 observers and replicates that found in Experiment 1.

The mean thresholds for the 6 observers are illustrated in Figure 6. Panels A and B show threshold-versus-set-size functions for one versus two targets, respectively. As with the example observers, there is a reversal in the magnitude of set-size effects. For onetarget tasks, the log-log slope of $0.32 \pm 0.08$ for identification is smaller than the log-log slope of $0.56 \pm 0.09$ for localization. For two-target tasks, the log-log slope of $0.57 \pm 0.11$ for identification is larger than the $\log -\log$ slope of $0.29 \pm 0.04$ for localization. This comparison is made explicit in Panel $\mathrm{C}$ of Figure 6. It plots
One Target

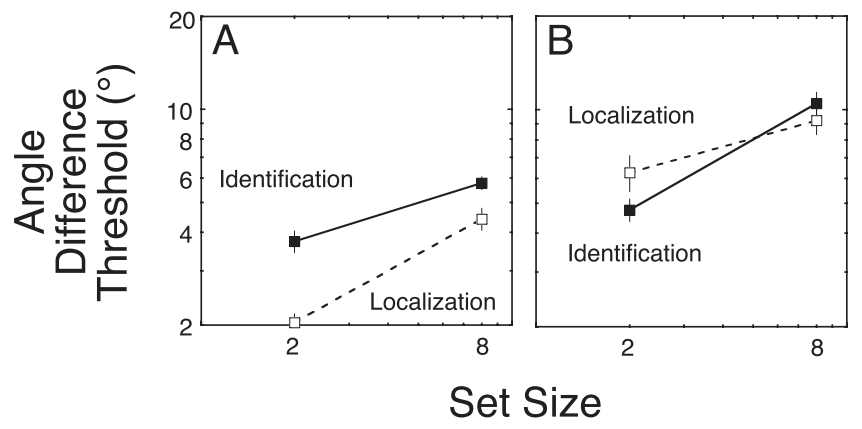

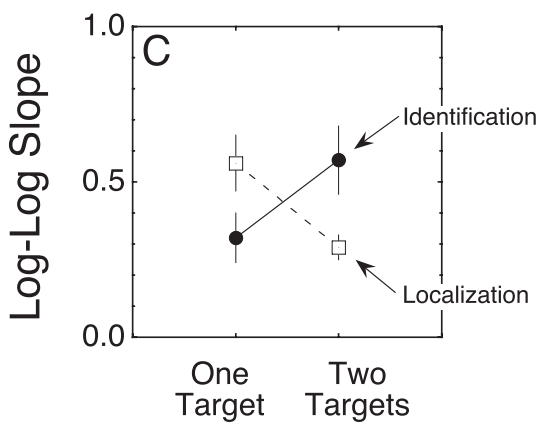

Figure 6. The results of Experiment 2 are summarized by the mean of estimates from all observers. The top two panels show that the set-size effects are larger for localization (dashed lines) for one target and larger for identification (solid lines) for two targets. The bottom panel summarizes this crossover interaction by plotting the log-log slope as a function of the number of targets. Error bars represent one standard error. 
the log-log slope for one- and two-target conditions. The solid symbols are for identification, and the open symbols are for localization. As with Experiment 1, there is a crossover interaction between the task and the number of targets. The interaction measure is $0.51 \pm 0.11$ and is reliable, $t(9)=4.5, p<.01$. Thus, the observed crossover interactions are very similar in Experiments 1 and 2.

\section{Discussion}

The privileged processing hypotheses predict that set-size effects depend on whether the task is identification or localization. The results of Experiment 1 showed that the ordering of set-size effects for identification and localization depended on the use of one versus two targets. The results of Experiment 2 generalize this result to $n$-alternative localization tasks. The threshold analysis yields the same result for both coarse and $n$-alternative localization. Thus, the evidence against the privileged processing hypothesis is not limited to coarse localization.

\section{Analysis 1: An Independent Channels Theory of Set-Size Effects}

The focus of this article is on demonstrating that set-size effects are not determined by the general distinction between identification and localization. Other specific features of the tasks matter. Here, we sketch a theory of these set-size effects based on signal detection theory by using independent channels for each stimulus. Details are in the Appendix. Somewhat to our surprise, the first theory we considered predicted the observed crossover interaction between number of targets and identification versus localization.

Signal detection theories of visual search have three kinds of assumptions. First, we assume that the relevant representations are noisy and correspond to single, real-valued random variables. Furthermore, for specific predictions we assume equal-variance Gaussian distributions. Second, we assume that the representations for each stimulus are independent. Two aspects of independence are relevant: unlimited capacity and statistical independence. For unlimited capacity, the quality of the representation of any one stimulus is independent of the number of the stimuli. For statistical independence, the value of the representation of any one stimulus is uncorrelated with another. Third, the two tasks require different decision rules. For yes-no identification, the observer is assumed to set an internal criterion and to respond "yes" if at least one of the items on the screen is tilted more than the criterion. For $n$-alternative localization, the observer is assumed to pick the location with the most evidence for a tilt to the left. Thus to compare these two tasks, we need a framework that builds in these different task demands.

The top panel of Figure 7 shows the representations for the two stimuli under target absent (left panels) and target present (right panels) trials. The abscissa specifies the stimulus representation, which is an internal transformation of angle, with target items appearing more tilted to the left. Thus, the target on average appears further to the right in the graphs of Figure 7. The distractors shown are Gaussian, and the numerical predictions also assume Gaussian distributions. However, the theory can make predictions from any reasonable distribution.
A

A
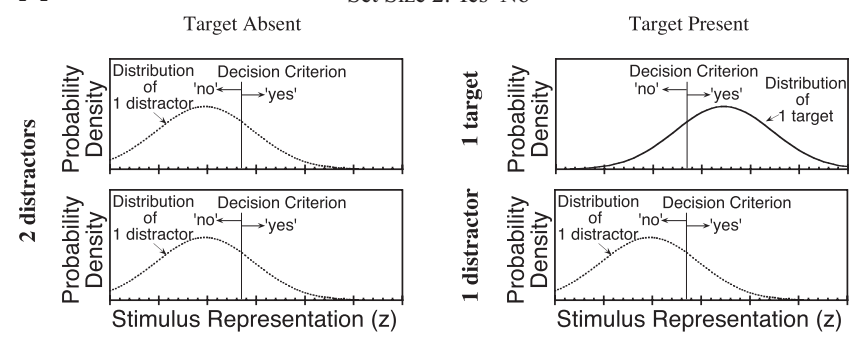

B
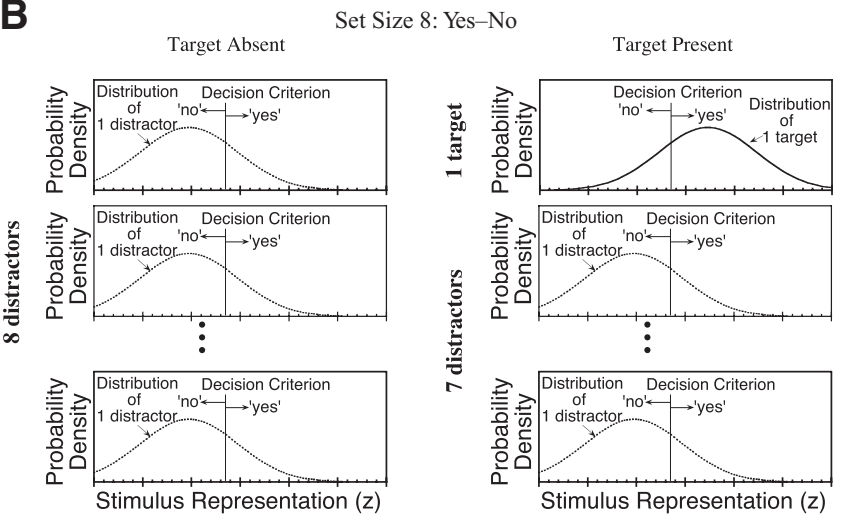

Figure 7. An illustration of the signal detection theory of set-size effects for yes-no identification. For set size 2 as illustrated in the top panels, a target absent trial has two opportunities to produce a false alarm. For set size 8 as illustrated by the bottom panels, a target absent trial has eight opportunities to produce a false alarm. The net result is reduced performance for set size 8 relative to set size 2 . This noisy integration process predicts a log-log slope on the threshold-versus-set-size graph of 0.22 . Targets are depicted with solid lines and distractors with dotted lines. The three vertical dots are placeholders for the five additional target absent graphs that are not shown due to space limitations.

For the yes-no identification task, we adopt a decision rule in which the observer makes a "yes" response if at least one stimulus item in the display exceeds an internal criterion. This rule is not quite ideal, although it is close. Thus on a target absent trial there are two samples from the target absent distribution, and a "yes" response is made if either exceeds the criterion.

The assumptions of unlimited capacity and statistical independence imply that as additional items are added to the display the processing of each individual item does not change. However, noisy integration can still produce an increase in threshold for larger set sizes. On a target absent trial for set size 2, the observer has two chances to make a false alarm. With set size 8 , the observer has eight chances to make a false alarm, as shown in the lower panels of Figure 7. To compensate and keep equal bias, the observer must increase the criterion for saying "yes," at the cost of reducing the hit rate. Thus this theory predicts a set-size effect even with unlimited capacity.

The $n$-alternative localization task differs in that a target is always present and the observer reports the location that is most likely to contain a target. Thus there is always one target and one distractor distribution for the signal detection model of the setsize- 2 condition for localization. For the ideal decision rule, the 
observer chooses the location with the most evidence of a target and reports it. Thus on a trial with set size 2, one samples from the two distributions and picks the location with the largest value. The observer makes an error if the distractor value exceeds the target value. With set size 8 , there are seven distractors, each of which might exceed the target item and lead to an error. There is now much greater overlap between the relevant distributions, leading to reduced performance and higher thresholds for set size 8 . Thus, the model for this task also produces set-size effects even with independent channels. Similar arguments are described for the twotarget tasks in the Appendix. These tasks introduce variations in the decision rule because of the need to distinguish the two targets in identification and the new ways to make an error in localization.

Here are the predictions for all four tasks in terms of the log-log slope comparing set size 2 with set size 8 . For one-target tasks, the predicted log-log slope is 0.22 for yes-no identification and 0.34 for coarse and $n$-alternative localization. In words, the set-size effects are predicted to be smaller for identification than for localization. For two-target tasks, the predicted log-log slope is 0.42 for two-target identification; 0.17 for two-target, coarse localization; and 0.14 for two-target, $n$-alternative localization. This reverses the order of the set-size effects for two-target tasks: Now, identification has larger set-size effects than does localization. Thus, the independent channel model predicts the observed crossover interaction. Some intuition for this prediction is developed at the end of the Appendix, but we offer a few highlights in the next section.

\section{Intuitions for Task Differences}

One of the most surprising aspects of the comparison between one- and two-target conditions is the difference observed at set size 2 in terms of performance in the localization task (see Figures $2 \mathrm{C}$ and $2 \mathrm{E}$ ). At first blush, these two conditions are quite similar: In both cases, the observer must identify the location of the tilted item among a number of distractors. However, at set size 2, adding an additional possible target increases the angle threshold by a factor of 5, while only doubling the threshold at set size 8 . One way to view these differences is that the two-target condition can be thought of as more like a set size 4, since the observer must track two possible targets. Thus it is handicapped from the start. However, these effects do not scale linearly, so that the differences between one- and two-target conditions become smaller at set size 8 .

A second source of the differences is the switch between a yes-no decision task and a two-alternative identification task. The latter can be employed with two targets but not one target. The analysis in the Appendix shows that even with two targets, a yes-no task yields smaller set-size effects than does a corresponding two-target identification task. One intuition for this effect is that when viewing a distractor there are more ways to go wrong with the two possible target choices rather than one "target" choice of "yes."

Not all is well. The predicted set-size effects are consistently smaller than those observed in Experiments 1 and 2, which may come, for example, from performance in set size 8 that is worse than the theory predicts based on set size 2. This decreased performance could result from a number of factors, and in the following two experiments and one new analysis, we explore the generality of the observed results and seek possible reasons for the discrepancy between the observed and predicted set-size effects.

\section{Experiment 3: Sensory Interactions in Space and Time}

Our analysis of Experiments 1 and 2 placed all the differences between the various tasks at the decision process and assumed a common perceptual representation across tasks. One test of this assumption is to vary the stimulus to see whether this affects the localization and identification tasks in different ways. We do this by manipulating both spatial frequency (by making the width of the bars in the grating smaller or larger) and temporal frequency (by flickering the stimuli on and off at several different flicker rates). If decision processing accounts for the task differences, then the results observed for one pair of spatial and temporal frequencies must generalize to other frequencies. In contrast, if perceptual processing differs for identification and localization, then spatial and temporal frequency manipulations may allow one to vary the mediating mechanism. In particular, our choice of manipulations is motivated by the idea that one can selectively stimulate visual pathways by using different combinations of spatial and temporal frequencies (e.g., Merigan \& Maunsell, 1993). While our manipulation may not isolate a particular pathway completely, it is likely to vary the contributions of the different pathways.

To test the spatial and temporal frequency dependencies of the two tasks, we measured the set-size effects at various combinations of spatial and temporal frequencies in yes-no identification and $n$-alternative localization as used in Experiment 2. In addition, to assess the dependence on contrast, we included a higher contrast condition for some combinations of spatial and temporal frequencies.

\section{Method}

The methods were similar to Experiment 2, with the manipulation of spatial frequency ( 1.5 or $3 \mathrm{cpd})$, and temporal frequency (2 or $18.75 \mathrm{~Hz}$ ), and contrast $(3 \mathrm{x}$ contrast threshold or $5 \mathrm{x}$ contrast threshold). The mean contrast value varied across flicker frequency and spatial frequency. For the $2-\mathrm{Hz}$ flicker, the mean contrasts across observers were $14 \%, 27 \%$, and $16 \%$ for the 1.5 cpd, 1.5 cpd higher contrast, and the 3 cpd conditions, respectively. For the $18.75-\mathrm{Hz}$ flicker, the values were $35 \%, 60 \%$, and $76 \%$, respectively. These values were identical for paired localization and identification tasks. The procedures were similar to Experiment 2, except that the temporal waveform of the stimulus was modulated either at $2 \mathrm{~Hz}$ or $18.75 \mathrm{~Hz}$. In order to accommodate the slower frequency, we increased the stimulus duration to $400 \mathrm{~ms}$. This modulation included the reversal of the grating (rather than modulating to gray). Four observers participated in the identification task first and then the localization task.

\section{Results}

Figure 8 shows the results of the various combinations of the three variables. To assess whether the three types of manipulations (spatial frequency, temporal frequency, and contrast) had any effect at all on the set-size effects for the two tasks, we performed a within-observers one-way analysis of variance for each task. For 
- Identification $\square$ Localization

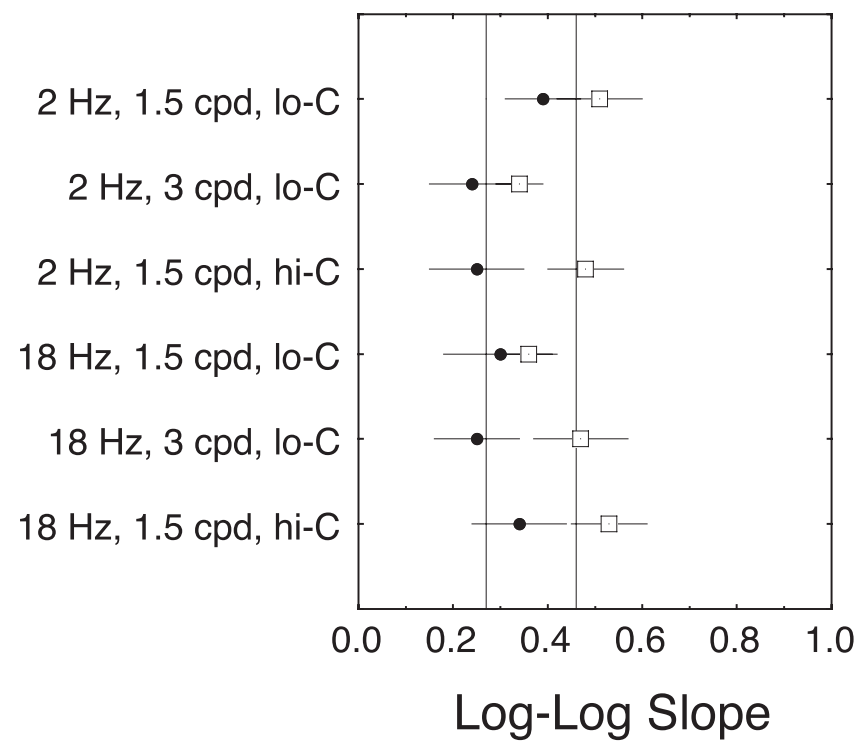

Figure 8. Set-size effects are summarized by the $\log$-log slope for all conditions in Experiment 3. Solid symbols represent identification tasks; open symbols represent localization tasks. The vertical lines show the means for the identification and localization tasks. $\mathrm{Hz}=$ cycles per second; cpd = cycles per degree; lo-C $=$ low contrast hi-C $=$ high contrast. Error bars represent one standard error.

both the yes-no identification and $n$-alternative localization tasks, no significant differences were found between any of the six conditions: identification, $F(5,15)<1$; localization, $F(5,15)=$ $1.65, p>.05$.

Without differences across spatial and temporal frequencies, we combined across conditions to produce an overall summary measure for the two tasks. The mean $\log -\log$ slope is $0.27 \pm 0.04$ for identification and $0.46 \pm 0.03$ for localization. These results replicate the one-target results of the Experiment 2. Thus, we can generalize our results to a range of spatial and temporal frequencies.

\section{Discussion}

On the basis of these results, we conclude that the identification and localization tasks do not show strong spatial and temporal frequency dependencies in our measure of set-size effects. This is consistent with similar processing of identification and localization. However, see Cameron et al. (2004) for possible limits on this generalization by using a larger range of spatial frequencies $(0.5$ to $12.0 \mathrm{cpd})$.

\section{Experiment 4: Cuing Control}

One possible explanation for finding set-size effects larger than predicted by the independent channel model is that sensory interactions may introduce dependencies for our displays. We deliberately tried to avoid sensory interactions by using widely spaced items: The nearest neighbor is a distance three times the width of a single stimulus item and more than half its eccentricity. In addition, we attempted to disrupt sensory interactions by randomizing the exact location of each stimulus and the phase of the gratings. However, it is possible that despite our efforts some kind of sensory interactions were present in our tasks. To test this, we conducted Experiment 4 with two conditions. The first was a control condition identical to the $n$-alternative localization task from Experiment 2. The second condition measured set-size effects by cuing a subset of stimuli in an otherwise identical display (see Palmer, 1994). For this relevant-set-size-2 case, the display had two pre-cues prior to the onset of the trial that indicated the relevant locations. The cues were always followed by eight stimuli, and the target appeared in one of the two cued locations. For relevant set size 8 , all eight locations were pre-cued. We ask whether the set-size effect differs between the control condition where the number of stimuli vary and the new condition where the number of stimuli are constant but a subset is cued. Effects of relevant set size that are smaller than of display set size are consistent with sensory interactions or ineffective cues. Effects of relevant set size that are larger than of display set size are consistent with phenomena such as crowding (Bouma, 1970; Pelli, Palomares, \& Majaj, 2004).

\section{Method}

The stimuli were identical to those in Experiment 2. The mean contrast value used across observers was $17 \%$ and was identical for cued and no-cued conditions for each observer. There were two conditions. One was identical to the $n$-alternative localization task of Experiment 2. The other always presented eight items on each trial, but for pre-cued locations with small plus signs prior to the start of the trial. The stimulus onset asynchrony between the cue and the stimuli was $800 \mathrm{~ms}$. The target then appeared in one of the two cued locations for $280 \mathrm{~ms}$ with a Gaussian temporal profile. The set-size- 8 condition used eight pre-cues in this task, with one target and seven distractors. Five observers participated.

\section{Results}

Table 1 contains the thresholds for set size 2 and 8 for the display-set-size and relevant-set-size conditions. Averaged across observers, there are no reliable differences. The display-set-size condition had a log-log slope of $0.47 \pm 0.07$, and the relevantset-size condition had a log-log slope of $0.43 \pm 0.10$. The magnitude of these set-size effects are similar to those found in the corresponding conditions of Experiments 2 and 3. Thus, this experiment demonstrates a similar set-size effect despite the use of

Table 1

Experiment 4: Set-Size Effects Using Cues or Varying the Number of Stimuli

\begin{tabular}{lcc}
\hline & \multicolumn{2}{c}{ Set size } \\
\cline { 2 - 3 } Condition & 2 & 8 \\
\hline No Cue & $2.5^{\circ} \pm 0.3^{\circ}$ & $4.8^{\circ} \pm 0.6^{\circ}$ \\
Cue & $2.3^{\circ} \pm 0.3^{\circ}$ & $4.2^{\circ} \pm 0.6^{\circ}$ \\
\hline
\end{tabular}

Note. Values are the orientation threshold in degrees. 

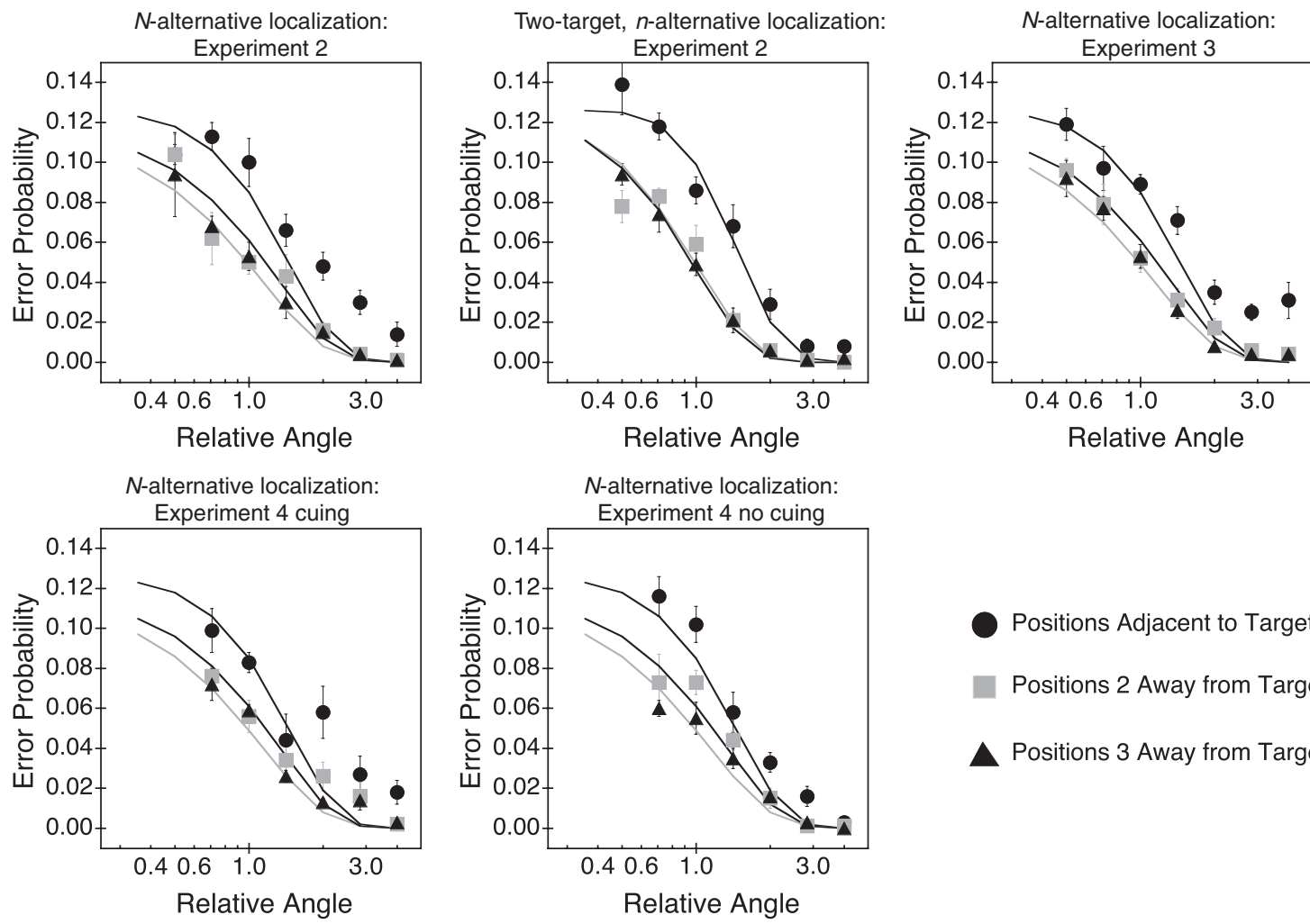

Positions Adjacent to Target

Positions 2 Away from Target

$\triangle$ Positions 3 Away from Target

Figure 9. Mislocalization data for set size 8 from Experiments 2, 3, and 4 aggregating over observers. The angle is defined relative to threshold performance for each observer. There are consistently more errors in the position adjacent to the target. Curves are the predictions of the overlapping channels model with one free parameter to describe the surplus of nearby mislocalizations. Error bars represent one standard error.

identical displays. Sensory interactions cannot be responsible for these effects of relevant set size.

\section{Analysis 2: Mislocalizations}

\section{Mislocalization Errors in Localization Tasks}

One possible explanation for the poor fit of the independent channel model is a failure of independent processing of the individual stimuli. Such a dependence can lead to mislocalizations such as found by Solomon and Morgan (2001). To measure the degree of mislocalization with set size 8 , we computed the errors for each position conditioned on how far away the position was from the target. The data for $n$-alternative localization in Experiments 2, 3, and 4 are shown in Figure 9. To combine across observers, we divided the six angles that were used to test each observer by that observer's threshold angle. This produced a relative angle, where 1.0 is the angle that produces approximate threshold performance ( $56 \%$ correct for set size 8 ), and smaller angles represent more difficult conditions. The angles used for each observer were then assigned to one of eight relative angles based on the angle's relation to the threshold angle for that observer in that condition. Performance at each relative angle was then averaged across observers to produce the graphs shown in Figure 9.

Positions adjacent to the target have systematically more errors than do locations two and three positions away from the target. The independent channel model does not predict this effect. One might propose that these mislocalizations come from confusions when translating a chosen location into a response on the keypad. However, two lines of evidence make this explanation implausible: In all of the set-size- 2 conditions, observers can make any of eight possible responses, but they made virtually no responses that do not specify one of the two stimuli (such responses are allowed and recorded as incorrect trials with respect to feedback). In addition, in the relevant-set-size-2 condition of Experiment 4, observers almost never made responses other than at one of the two pre-cued locations. Thus, the basis for the mislocalizations is probably not response confusion on the part of the observers. Next, we consider an alternative explanation for mislocalizations.

\section{The Overlapping Channels Model}

One account of mislocalizations (Parkes, Lund, Angelucci, Solomon, \& Morgan, 2001) is that observers pool information from nearby stimuli. We use this pooling idea as the basis of our overlapping channel model. In it, we relax the assumption of stochastic independence and replace it with a weighted pooling among neighboring stimuli. This is similar to models of crowding (e.g., Pelli et al., 2004) and the interactive channel model (Estes, 1982). Our approach is to extend the model with one additional free parameter to account for the mislocalization data and then test its prediction of the set-size effect. 
In the overlapping channels model, one assumes that on a given trial the representations of the two or eight stimuli are obtained with unlimited-capacity processing. In other words, increasing the number of items does not change the quality of the information at each location. However, the representation is dependent on its neighbors in another way. The interaction is by pooling, in which the values of the representations associated with each stimulus blend together. We call this an overlapping channel model to distinguish it from the more specific independent channels model or the more general interactive channel model.

The lack of independence implied by pooling changes the expected set-size effects. Because the pooling process is only relevant to the set-size- 8 condition, the overlapping channel model predicts an increase of the set-size effect relative to independent processing (see the Appendix for details).

By using weights estimated from the mislocalization data, we derived the predicted set-size effects for each task. As before, the predictions are for the log-log slope comparing set size 2 with set size 8 . For one-target tasks, the predicted log-log slope is 0.28 for yes-no identification, 0.37 for coarse localization, and 0.40 for $n$-alternative localization. For two-target tasks, the predicted loglog slope is 0.47 for two-target identification; 0.36 for two-target, coarse localization; and 0.42 for two-target, $n$-alternative localization. Importantly, these predictions maintain the crossover interaction and are closer to the effects observed in the experiments. The point here is that a modest alteration of the independent channels model can predict the details of the observed crossover interaction.

\section{Limited Capacity and Overlapping Channels}

The overlapping channels model breaks the independence of the stimuli at the level of the initial sensory representation. This kind of dependency that produces correlated inputs places limitations on capacity when the system is considered as a whole, as did both Broadbent (1958) and Estes (1982). However, others use the term limited capacity to refer to the dependency of the processing of individual items on the presence or absence of other items (Townsend, 1974). By this definition, the overlapping channels model has unlimited capacity. Note that the presence of set-size effects alone is not diagnostic for limited-capacity processing (e.g., Palmer et al., 1993; Thornton \& Gilden, 2007; Townsend, 1974). In summary, whether one characterizes the overlapping channels model as having unlimited or limited capacity depends on one's taste: specifically, whether one focuses on the processing of individual stimuli or the entire system.

\section{General Discussion}

We measured set-size effects for identification and localization for several different accuracy tasks. For the one-target tasks, set-size effects were smaller for identification. In contrast, for two-target tasks, set-size effects were smaller for localization. This reversal was predicted by an independent channels model based on signal detection theory. Moreover, the reversal makes clear that there is nothing about identification or localization per se that results in smaller set-size effects. The reversal is inconsistent with the privileged processing hypothesis.
Additional experiments and analyses further constrain theories of identification and localization. For example, equating the number of responses using a coarse localization task did not change the pattern of results. More quantitatively, a closer look at the errors in localization reveals a pattern of nearby mislocalizations that can be attributed to pooling between neighboring stimuli (e.g., Morgan, Ward, \& Castet, 1998; Solomon \& Morgan, 2001). The independent channels model can be modified by including pooling between channels. This overlapping channels model does better at predicting the magnitude of the set-size effects reported here. Thus, these results are consistent with similar processing for identification and localization.

\section{Current Status of Similar Processing and Close Binding}

In this article, we find evidence from set-size effects that is consistent with similar processing for identification and localization. In the next section, we put this result into the context of other ways to distinguish similar versus privileged processing of identity versus location. Is our result isolated or common?

Relation to response time studies. Are the results found here for identification and localization general to response time? Even if this question is restricted to response time tasks for singlefixation search, it is difficult to answer. Nothing in the existing response time studies compares identification and localization with either the range of tasks (one vs. two targets) of the current study or the highly controlled two-by-two paradigm of Marc Green (1992). Moreover, most response time experiments do not control critical factors that influence the magnitude of set-size effects. Perhaps the most important of these is target-distractor discriminability (e.g., Duncan \& Humphreys, 1989). In the current study, the interaction of set size and target-distractor discriminability was measured and characterized for each task by the thresholdversus-set-size function.

To begin comparing identification and localization with response time tasks, at least two things need to be done. First, the interaction between set size and target-distractor discriminability must be measured in the context of a response time task. One early effort to do this was Palmer (1998), which found this interaction to be quite similar for accuracy and response time if the other details of the experiment are kept constant (e.g., sparse displays, common stimulus values, etc.). Second, the theory of signal detection as applied to visual search must be generalized to response time tasks. The first part of this generalization is to adapt sequential sampling theories that can describe both accuracy and response time experiments (for a general review, see Luce, 1986; for a review of the application to search, see Palmer et al., 2000). A recent article by Thornton and Gilden (2007) is a nice example of applying these ideas to the distinction between serial and parallel processes in visual search. It remains for researchers to use these ideas to compare identification and localization tasks.

Relative performance of localization and identification. Perhaps the simplest comparison of identification and localization is a direct comparison of performance (Atkinson \& Braddick, 1989). Perhaps the best-to-date comparison of this kind is by M. Green (1992). He used a two-by-two task with two display intervals with varying orientation. One response was a two-interval forced choice (2IFC) identification judgment (called detection in the article), and the other was a coarse left-right localization judgment. With this 
combination of tasks, performance was essentially identical for the two tasks. M. Green argued that this particular pair of tasks should result in identical performance, while other parings need not be identical in performance. Similar analyses can be found in Solomon and Morgan (2001).

In our experiments, one can also measure the localizationidentification threshold ratio. Consider the set-size- 2 conditions to make the analysis the most distinct from the set-size analysis already presented. For the one-target tasks, the observed ratio was $0.54 \pm 0.03$ in Experiment 1 and $0.56 \pm 0.03$ in Experiment 2. The prediction was 0.5 . For the two-target tasks, the observed ratio was $1.5 \pm 0.1$ in Experiment 1 and $1.4 \pm 0.3$ in Experiment 2. The prediction was 1.6. Thus, in all four cases the observed ratios are near the predictions. Moreover, the ratios reverse between the oneand two-target tasks just as did the relative magnitude of the set-size effects. This additional bit of evidence is consistent with the decision structure of the tasks determining performance rather than identity versus location, per se.

Conditional performance of identification and localization. Perhaps the best-known approach to the relation between identification and localization are estimates of the dependency between these judgments when they are both made on a single trial. Specifically, one estimates the probability of identification given no location information P(I | no L) or the probability of localization given no identity information $\mathrm{P}(\mathrm{L} \mid$ no I). Close binding predicts both of these conditional probabilities are zero. The localizationwithout-identification and identification-without-localization hypotheses predict one or the other probability is above chance. In the extreme, if identification and localization are independent, then the conditional probability is equal to the unconditional probabilities. Early experiments have found support for identification without localization (e.g., Treisman \& Gelade, 1980) and for localization without identification (e.g., Müller \& Rabbitt, 1989).

Johnston and Pashler (1990) have identified several ways the conditional probabilities can be influenced by the details of the tasks. They control for these effects and were able to account for much if not all of the observed conditional probability. They find that the $\mathrm{P}(\mathrm{I} \mid$ no $\mathrm{L})$ is near zero, which is inconsistent with identification without localization. In addition, they find that $\mathrm{P}(\mathrm{L}$ । no I) was around .1 in the context of correct localization probabilities of .75 . Since then, several investigations have further pursued this approach. For example, van der Heijden and colleagues have investigated a single stimulus task with two- or four-choice judgments of orientation and color paired with two- or four-choice judgments of location (van der Heijden, Wolters, \& Brouwer, 1995; Bloem \& van der Heijden, 1995). In some tasks, they found $\mathrm{P}(\mathrm{I} \mid \mathrm{no} \mathrm{L})$ above zero but also found evidence for the negative information problem. In their best-controlled study, they found $\mathrm{P}(\mathrm{I} \mid$ no L) near zero. They concluded that there is no evidence of identification without localization. In summary, several recent studies have found no evidence for identification without localization and some evidence for localization without identification. Thus, the bulk of the evidence is consistent with close, but not perfect, binding of identity and location.

Illusory conjunctions. The identification-without-localization hypothesis has generated an investigation into relations between judgments of multiple features. The idea is that if identification of features can occur without localization, then different features such as color and orientation can be miscombined. Treisman and
Schmidt (1982) have estimated the probability of such illusions by comparing the relative frequency of feature errors versus conjunction errors. Consider a display with a red $\mathrm{O}$ and a green $\mathrm{X}$. The report of a blue $\mathrm{X}$ is a feature error while the report of a red $\mathrm{X}$ is a conjunction error. Several experiments have found that conjunction errors are more frequent than feature errors (Treisman \& Schmidt, 1982; for reviews, see Prinzmetal, 1995; Tsal, 1989a, 1989b).

Interpreting this result requires addressing all of the issues introduced by Johnston and Pashler (1990). Here, we focus on one particular issue that has received the most attention: Are the conjunction errors due to a post-perceptual process such as guessing rather than a conjunction in perception (Ashby, Prinzmetal, Ivry, \& Maddox, 1996; Navon \& Ehrlich, 1995)? If conjunction responses are due to guessing, then they do not support the hypothesis of identification without localization. For example, suppose two stimuli are presented; one stimulus is cued with a bar marker, and the observer reports the color and orientation of that stimulus. Ashby et al. (1996) have devised a multinomial model to predict the pattern of errors observed in this task. It includes parameters for the discrimination of each feature and for the probability of correctly binding the features in perception. They find that the results are best described when the binding probability was high but less than one. Another possibility explored by Donk (1999) is that target/non-target confusions make up the complete set of conjunction errors that have been attributed to an illusory conjunction mechanism. If the cue was mislocalized or in some other way causes the selection of the wrong stimulus, then such errors may inflate the estimate of the binding probability. She has conducted a series of experiments that extended the experiments of Ashby and colleagues by adding selection criteria that allow one to distinguish failures of selection from failures of binding. By using this more general decision model with a parameter for selection errors, she finds evidence for imperfect selection and perfect binding. While this interpretation is subject to a continuing debate (Donk, 2001; Prinzmetal, Diedrichsen, \& Ivry, 2001), the analysis is a step forward and demonstrates the need for formal models to isolate different processes. It is an open question of whether the mislocalizations found in the current data are related to processes that may generate illusory conjunctions.

This review has shown that three additional lines of behavioral evidence are largely consistent with similar processing and close binding of identity and location. Interestingly, one can also find critical treatments of the neuroscience evidence against close binding of identity and location information (e.g., Ghose \& Maunsell, 1999; Reynolds \& Desimone, 1999; Rossetti, Pisella, \& Vighetto, 2003). Thus, while there are interesting phenomena relevant to comparisons between identification and localization, the evidence against similar processing and close binding is not definitive. Our study further undermines one of the arguments against similar processing and close binding: Set-size effects do not depend on identification versus localization per se.

\section{Conclusions}

Prior studies have shown large differences in set-size effects for identification and localization tasks. We show that these effects are specific to the details of the tasks and not specific to identification versus localization. Thus, these effects do not support hypotheses 
specifying privileged processing of either identity or location. Instead, we find that accuracy search tasks yield results consistent with similar processing of identity or location. We presented an example theory of such similar processing by using a model that was tailored to the decision structure of each task and allowed pooling between nearby channels.

\section{References}

Ashby, F. G., Prinzmetal, W., Ivry, R., \& Maddox, W. T. (1996). A formal theory of feature binding in object perception. Psychological Review, 103(1), 165-192.

Atkinson, J., \& Braddick, O. J. (1989). "Where" and "what" in visual search. Perception, 18, 181-189.

Baldassi, S., \& Burr, D. C. (2000). Feature-based integration of orientation signals in visual search. Vision Research, 40, 1293-1300.

Baldassi, S., \& Verghese, P. (2001). Comparing integration rules in visual search. Journal of Vision, 2, 559-570.

Bennett, P., \& Jaye, P. (1995). Letter localization, not discrimination is constrained by attention. Canadian Journal of Experimental Psychology, 49, 460-503.

Bloem, W., \& van der Heijden, H. A. C. (1995). Complete dependence of color identification upon color localization in a single-item task. Acta Psychologica, 89, 101-120.

Bouma, J. (1970, April 11). Interaction effects in parafoveal letter recognition. Nature, 226, 177-178.

Broadbent, D. E. (1958). Perception and communication. London: Pergamon.

Burgess, A. E., \& Ghandeharian, H. (1984). Visual signal detection: II. Signal-location identification. Journal of the Optical Society of America A, 1, 906-910.

Cameron, E. L., Tai, J. C., Eckstein, M. P., \& Carrasco, M. (2004). Signal detection theory applied to three visual search tasks-identification, yes/no detection and localization. Spatial Vision, 17, 295-326.

Creelman, C. D., \& Macmillan, N. A. (1979). Auditory phase and frequency discrimination: A comparison of nine procedures. Journal of Experimental Psychology: Human Perception and Performance, 5, 146-156.

Dobkins, K. R., \& Bosworth, R. G. (2001). Effects of set-size and selective spatial attention on motion processing. Vision Research, 41, 1501-1517.

Donk, M. (1999). Illusory conjunctions are an illusion: The effects of target-nontarget similarity on conjunction and feature errors. Journal of Experimental Psychology: Human Perception and Performance, 25, $1207-1233$

Donk, M. (2001). Illusory conjunctions die hard: A reply to Prinzmetal, Diedrichsen, and Ivry (2001). Journal of Experimental Psychology: Human Perception and Performance, 27, 542-546.

Donk, M., \& Meinecke, C. (2001). Feature localization and identification. Acta Psychologica, 160, 97-119.

Duncan, J., \& Humphreys, G. W. (1989). Visual search and stimulus similarity. Psychological Review, 96, 433-458.

Eckstein, M. P., \& Whiting, J. S. (1996). Visual signal detection in structured backgrounds: I. Effect of number of possible spatial locations and signal contrast. Journal of the Optical Society of America A, 13, $1777-1787$.

Estes, W. K. (1982). Similarity-related channel interactions in visual processing. Journal of Experimental Psychology: Human Perception and Performance, 8, 353-382.

Foley, J. M., \& Schwarz, W. (1998). Spatial attention: Effect of position uncertainty and number of distractor patterns on the threshold-versuscontrast function for contrast discrimination. Journal of the Optical Society of America A, 15, 1036-1047.

Ghose, G. M., \& Maunsell, J. (1999). Specialized representations in visual cortex: A role for binding? Neuron, 24, 79-85.
Graham, N. (1989). Visual pattern analyzers. New York: Oxford University Press.

Green, D. M., \& Luce, R. D. (1975). Theoretical note: Parallel psychometric functions from a set of independent detectors. Psychological Review, 82, 483-486.

Green, D. M., \& Swets, J. A. (1966). Signal detection theory and psychophysics. New York: Krieger.

Green, M. (1992). Visual search: Detection, identification, and localization. Perception, 21, 765-777.

Haxby, J. V., Horwitz, B., Ungerleider, L. G., Maisog, J. M., Pietrini, P., \& Grady, C. L. (1994). The functional organization of human extrastriate cortex: A PET-rCBF study of selective attention to faces and locations. The Journal of Neuroscience, 14, 6336-6353.

Hoffman, J. E. (1979). A two-stage model of visual search. Perception \& Psychophysics, 25, 319-327.

Irwin, D. E., \& Brown, J. S. (1987). Tests of a model of informational persistence. Canadian Journal of Experimental Psychology, 41, $317-$ 338.

Johnston, J. C., \& Pashler, H. (1990). Close binding of identity and location in visual feature perception. Journal of Experimental Psychology: Human Perception and Performance, 16, 843-856.

Klein, S. (1985). Double-judgment psychophysics. Journal of the Optical Society of America A, 2, 1560-1585.

Lee, C., \& Estes, W. K. (1981). Item and order information in short-term memory: Evidence for multilevel perturbation processes. Journal of Experimental Psychology: Human Learning and Memory, 7, 149-169.

Loftus, G. R., Oberg, M. A., \& Dillon, A. M. (2004). Linear theory, dimensional theory, and the face-inversion effect. Psychological Review, $111,835-863$.

Luce, R. D. (1986). Response times. New York: Oxford University Press.

Merigan, W. H., \& Maunsell, J. H. R. (1993). How parallel are the primate visual pathways? Annual Review of Neuroscience, 16, 369-402.

Mewhort, D. J., Campbell, A. J., Marchetti, F. M., \& Campbell, J. I. (1981). Identification, localization, and "iconic memory": An evaluation of the bar-probe task. Memory \& Cognition, 9, 50-67.

Mewhort, D. J. K., Huntley, M. F., \& Duff-Fraser, H. (1993). Masking disrupts recovery of location information. Perception \& Psychophysics, $54,759-762$

Monnier, P., \& Nagy, A. (2001). Set-size and chromatic uncertainty in an accuracy visual search task. Vision Research, 41, 3817-3827.

Morgan, M. J., Ward, R. M., \& Castet, E. (1998). Visual search for a tilted target: Tests of spatial uncertainty models. The Quarterly Journal of Experimental Psychology, 51A, 347-370.

Müller, H. J., \& Rabbitt, P. M. (1989). Reflexive and voluntary orienting of visual attention: Time course of activation and resistance to interruption. Journal of Experimental Psychology: Human Perception and Performance, 15, 315-330.

Nachmias, J., \& Kocher, E. C. (1970). Visual detection and discrimination of luminance increments. Journal of the Optical Society of America, 60, 382-389.

Navon, D., \& Ehrlich, B. (1995). Illusory conjunctions: Does inattention really matter? Cognitive Psychology, 29, 59-83.

Nothdurft, H. C. (1985). Sensitivity for structure gradient in texture discrimination tasks. Vision Research, 25, 1957-1968.

Nothdurft, H. C. (1991). Texture segmentation and pop-out from orientation contrast. Vision Research, 31, 1073-1078.

Nothdurft, H. C. (1992). Feature analysis and the role of similarity in preattentive vision. Perception \& Psychophysics, 52, 355-375.

Nothdurft, H. C. (1993). The role of features in preattentive vision: Comparison of orientation, motion and color cues. Vision Research, 33, 1937-1958.

Palmer, J. (1994). Set-size effects in visual search: The effect of attention is independent of the stimulus for simple tasks. Vision Research, 34, $1703-1721$. 
Palmer, J. (1995). Attention in visual search: Distinguishing four causes of set-size effects. Current Directions in Psychological Science, 4, 118 123.

Palmer, J. (1998). Attentional effects in visual search: Relating search accuracy and search time. In R. D. Wright (Ed.), Visual attention (pp. 384-388). New York: Oxford University Press.

Palmer, J., Ames, C. T., \& Lindsey, D. T. (1993). Measuring the effect of attention on simple visual search. Journal of Experimental Psychology: Human Perception and Performance, 19, 108-130.

Palmer, J., Verghese, P., \& Pavel, M. (2000). The psychophysics of visual search. Vision Research, 40, 1227-1268.

Parkes, L., Lund, J., Angelucci, A., Solomon, J. A., \& Morgan, M. (2001). Compulsory averaging of crowded orientation signals in human vision. Nature Neuroscience, 4, 739-744.

Pelli, D. G. (1987). On the relation between summation and facilitation. Vision Research, 27, 119-123.

Pelli, D. G. (1997). The VideoToolbox software for visual psychophysics: Transforming numbers into movies. Spatial Vision, 10, 437-442.

Pelli, D. G., Palomares, M., \& Majaj, N. J. (2004). Crowding is unlike ordinary masking: Distinguishing feature integration from detection. Journal of Vision, 4, 1136-1169.

Prinzmetal, W. (1995). Visual feature integration in a world of objects. Current Directions in Psychological Science, 4, 90-94.

Prinzmetal, W., Diedrichsen, J., \& Ivry, R. B. (2001). Illusory conjunctions are alive and well: A reply to Donk (1999). Journal of Experimental Psychology: Human Perception and Performance, 27, 538-541.

Quick, R. F. (1974). A vector-magnitude model of contrast detection. Kybernetik, 16, 65-67.

Quinlan, P. T. (2003). Visual feature integration theory: Past, present, and future. Psychological Bulletin, 129, 643-673.

Reynolds, J. H., \& Desimone, R. (1999). The role of neural mechanisms of attention in solving the binding problem. Neuron, 24, 19-29.

Rossetti, Y., Pisella, L., \& Vighetto, A. (2003). Optic ataxia revisited: Visually guided action versus immediate visuomotor control. Experimental Brain Research, 153, 171-179.

Saarinen, J. (1996). Localization and discrimination of "pop-out" targets. Vision Research, 36, 313-316.

Sagi, D., \& Julesz, B. (1985a). Detection versus discrimination of visual orientation. Perception, 14, 619-628.

Sagi, D., \& Julesz, B. (1985b, June 7). "Where" and "what" in vision. Science, 228, 1217-1219.

Shaw, M. L. (1980). Identifying attentional and decision-making components in information processing. In R. S. Nickerson (Ed.), Attention and performance VIII (pp. 106-121). Hillsdale, NJ: Erlbaum.
Solomon, J. A., \& Morgan, M. (2001). Odd-men-out are poorly localized in brief exposures. Journal of Vision, 1, 9-17.

Swensson, R. G., \& Judy, P. F. (1981). Detection of noisy visual targets: Models for the effects of spatial uncertainty and signal-to-noise ratio. Perception \& Psychophysics, 29, 521-534.

Tanner, W. P., Jr. (1961). Physiological implications of psychophysical data. Annals of the New York Academy of Science, 89, 752-765.

Thomas, J. P. (1985). Detection and identification: How are they related? Journal of the Optical Society of America A, 2, 1457-1467.

Thornton, T. L., \& Gilden, D. L. (2007). Parallel and serial processes in visual search. Psychological Review, 114(1), 71-103.

Townsend, J. T. (1974). Issues and models concerning the processing of a finite number of inputs. In B. H. Kantowitz (Ed.), Human information processing: Tutorials in performance and cognition (pp. 133-168). Hillsdale, NJ: Erlbaum.

Treisman, A., \& Gelade, G. (1980). A feature-integration theory of attention. Cognitive Psychology, 12, 97-136.

Treisman, A., \& Gormican, S. (1988). Feature analysis in early vision: Evidence from search asymmetries. Psychological Review, 95, 15-48.

Treisman, A., \& Schmidt, H. (1982). Illusory conjunctions in the perception of objects. Cognitive Psychology, 14, 107-141.

Tsal, Y. (1989a). Do illusory conjunctions support the feature integration theory? A critical review of theory and findings. Journal of Experimental Psychology: Human Perception and Performance, 15, 394-400.

Tsal, Y. (1989b). Further comments on feature integration: A reply to Briand and Klein. Journal of Experimental Psychology: Human Perception and Performance, 15, 407-410.

Ungerleider, L. G., \& Mishkin, M. (1982). Two cortical visual systems. In D. J. Ingle, M. A. Goodale, \& R. J. W. Mansfield (Eds.), Analysis of visual behavior (pp. 549-586). Cambridge, MA: MIT Press.

van der Heijden, A. H. C., Wolters, G., \& Brouwer, R. F. T. (1995). Response dependency and processing dependency of line orientation and position in a single-item task. Psychological Research/Psychologische Forschung, 58, 19-30.

van Essen, D. C., \& Gallant, J. L. (1994). Neural mechanisms of form and motion processing in the primate visual system. Neuron, 13, 1-10.

Watson, A. B., \& Pelli, D. G. (1983). QUEST: A Bayesian adaptive psychometric method. Perception \& Psychophysics, 33, 113-120.

Wolford, G. (1975). Perturbation model for letter identification. Psychological Review, 82, 184-199.

Wolford, G., \& Shum, K. (1980). Evidence for feature perturbations. Perception \& Psychophysics, 27, 409-420. 


\section{Appendix}

\section{Derivation of Localization and Indentification Models From Signal Detection Theory}

In this Appendix, we derive the predictions of the independent channel model for a variety of tasks. These predictions are developed for arbitrary set sizes, but the numerical examples use set sizes 2 and 8 . We also describe the predictions of the overlapping channels model and provide intuitions about why the predictions differ across task.

\section{Common Notation and Assumptions}

We follow the notation commonly used in signal detection theory (D. M. Green \& Swets, 1966; for more detail, see Palmer et al., 2000). The representation of each stimulus corresponds to a real-valued random variable. For targets and distractors, these random variables are designated $T$ and $D$, respectively. Random variables for $n$ distractors are subscripted $D_{l}, \ldots, D_{n}$. We assume two kinds of independence. First, for all of the models, assume unlimited capacity in the processing of the relevant representations. Hence, the mean and variance for any $T$ or $D$ random variable are independent of the set size. Second, for all but the overlapping channels model, assume statistical independence between the random variables. On a particular trial, the value of any random variable, say $D_{l}$, is independent of the value of any other representation on that same trial, say $D_{2}$.

We further assume that all the distractor random variables $D_{i}$ are identically distributed with a density function of $f_{i}$ and a cumulative distribution function of $F_{i}$. In addition, targets are represented by a shift family of the same distribution. If the distractor density is $f(x)$, the target density is $f(x-w s)$, where $s$ is the physical description of the target distractor difference, and $w$ is a free parameter relating physical units to the units of the internal representation (e.g., $\left.d^{\prime}\right)$. Finally, for all specific numerical calculations, make the further assumption that all random variables are Gaussian.

\section{Yes-No Identification}

The analysis of set-size effects for yes-no tasks dates to Tanner (1961), and a recent derivation of the effect on threshold can be found in Palmer et al. (1993). Performance on target present and target absent trials is characterized by the hits and false alarms, respectively. Assume that each stimulus engenders an internal response that is compared with a criterion $c$. Further assume that the observer indicates "target present" if at least one stimulus representation has a value above the criterion $c$.

First, consider the distributions associated with a single stimulus item. For a single distractor, the probability of a false alarm is

$$
p(F A)=p(D>c),
$$

which is equal to

$$
=1-F(c) .
$$

Similarly, the probability of a hit is

$$
p(\text { hit })=p(T>c),
$$

which is equal to

$$
=1-F(c-w s) .
$$

For $n$ items, where $n$ is greater than 1 , one has

$$
\begin{gathered}
p(\mathrm{FA})=p\left(D_{1}, D_{2}, \ldots, \text { or } D_{n}>c\right) \\
=1-F(c)^{n} .
\end{gathered}
$$

With one target and $n-1$ distractors, one has

$p$ (hit I 1 target, $n-1$ distractors)

$$
=p \text { (“at least one above criterion"), }
$$

which is equal to

$$
=1-F(c-w s) F(c)^{n-1} .
$$

This equation computes the probability of a miss of the target times the probability of correct rejections for all $n-1$ distractors. One minus this product is the probability of a hit. A correct response on a target present trial can result from either a target or a distractor exceeding the criterion. In fact, the distractors contribution occurs more and more as the set size $n$ increases. Of course, this effect also occurs on target absent trials, and observers must adjust their criterion accordingly to prevent more and more false alarms. We assume that observers adjust their criterion in the set-size- 2 and set-size- 8 blocks so that they have equal bias, $P($ hit $)=P($ correct rejection $)$.

To solve these equations to find a stimulus threshold, assume that observers have equal bias, and solve for the unknowns $c, w$, and $s$. The goal is to predict the change in threshold sensitivity as a function of set size. Thus, although one cannot measure $s$ directly, one can measure the threshold sensitivity as a function of set size, which is $s_{t}(n)$.

The next step is to solve for $s_{t}(n)$. To do this, let the criterion false alarm probability be $k=.25$, and the criterion hit probability for threshold be $1-k=.75$. Setting Equations A3 and A4 equal to these values and substituting $s_{t}(n)$ for $s$ in Equation A4, one obtains

$$
k=1-F(c)^{n}
$$

and

$$
k=F\left(c-w s_{t}(n)\right) F(c)^{n-1} .
$$

The parameter $w$ is a free parameter that indicates the sensitivity scaling for each observer. The next step is to solve for $c$ from Equation A5,

$$
\begin{aligned}
& F(c)=(1-k)^{1 / n}, \\
& c=F^{-1}\left[(1-k)^{1 / n}\right],
\end{aligned}
$$

and then use this to solve for $s_{t}(n)$ by substitution with Equation A6 (see Palmer, 1998),

$$
s_{t}(n)=(1 / w)\left\{F^{-1}\left[(1-k)^{1 / n}\right]-F^{-1}\left[k /(1-k)^{(n-1) / n}\right]\right\},
$$


where $F^{-1}$ represents the inverse cumulative function. Note that there still is the free parameter $w$ which represents sensitivity. Rather than fit this parameter, instead consider the ratio of two set sizes, which causes both $w$ values to cancel. By convention, we express this ratio by the log-log slope, which equals

$$
\alpha=\frac{\log \left(s_{t}\left(n_{2}\right)\right)-\log \left(s_{t}\left(n_{1}\right)\right)}{\log \left(n_{2}\right)-\log \left(n_{1}\right)},
$$

where $\alpha$ is the set-size effect expressed as a ratio of log differences (a ratio of ratios), $n_{1}$ and $n_{2}$ are the first and second set-size values used (e.g., two and eight in the present study), and $s_{t}(n)$ is the stimulus threshold for set-size $n$.

Assuming Gaussian distributions, a criterion of $k=.25$ and $1-$ $k=.75$ for false alarms and hits, the predicted log-log slope is 0.22 for these set sizes.

\section{$N$-Alternative Localization}

Here we follow the analysis of the $n$-alternative localization task of Shaw (1980). As with identification, the stimulus is onedimensional. For localization, assume that the observer chooses the location of the stimulus with the largest value in the relevant dimension (e.g., leans to the left the most). This is equivalent to a max rule on an appropriately standardized representation. For set size $n$, the probability of choosing the correct location is

$$
p(\text { correct })=p\left[T>\max \left(D_{1}, \ldots D_{n-1}\right)\right],
$$

which is equal to

$$
=\int_{-\infty}^{\infty} f(x-w s) F(x)^{n-1} d x .
$$

This equation integrates the product of a shifted density function representing the target distribution, multiplied by the cumulative distribution representing the distractor distribution(s).

To find a threshold stimulus, assume $w=1$ and use a numerical procedure to find the $s$ value that produces $75 \%$ correct for $n=2$ and produces $56.25 \%$ correct for $n=8$. With the log-log slope formula (Equation A9) and assuming Gaussian distributions, the predicted $\log -\log$ slope is 0.34 , which is higher than the 0.22 for yes-no.

\section{Coarse Localization}

In coarse localization, the target item is grouped with $n / 2-1$ distractors on one side, and there are $n / 2$ distractors on the other side. Observers indicate which side of the display contains the target. Observers can get the right answer accidentally by erroneously believing that one of the distractors on the target side was the target. If they choose one of the distractors on the side opposite the target, they get the wrong answer. The probability of selecting the correct side is

$$
p(\text { correct })=\max \left(T, D_{1}, \ldots D_{n / 2-1}\right)>\max \left(D_{n / 2}, \ldots D_{n-1}\right),
$$

which is equal to

$$
=1-\frac{n}{2} \int_{-\infty}^{\infty} f(x) F(x-w s) F(x)^{n-2} d x .
$$

As with $n$-alternative localization, to find a stimulus threshold, a numerical procedure is used to find the value $s_{t}$ that produces $75 \%$ correct for both $n=2$ and $n=8$. Assuming Gaussian distributions and set sizes 2 and 8 , the predicted $\log -\log$ slope is 0.34 , identical to the $n$-alternative localization value and higher than the 0.22 value for yes-no. The identity in predictions for different localization tasks motivates our use of different thresholds in the localization tasks.

\section{Two-Target Identification}

Here, we follow the analyses of Solomon and Morgan (2001) and Baldassi and Verghese (2001). In our example of two-target identification, the target item is either a left-leaning or rightleaning grating, and the distractors are all vertical. Two responses are possible on each trial, called the "larger" response and the "smaller" response. Define the target deviating from the distractors in a positive direction as $T$ and the target deviating in a negative direction $T^{\prime}$. Assume the observer chooses the response corresponding to the evidence that deviates the most from that for the median distractor, which is defined as zero. Suppose that a larger target is presented on a trial. Two events lead to a correct response of "larger." First, the target item may yield the most evidence of deviation and deviate in the positive direction. Second, a distractor item may yield the most evidence of deviation, and deviate in a positive direction, which also leads to the correct answer (but for the wrong reason).

Thus the probability of correctly saying "larger" if $T$ is presented on the trial equals

$$
\begin{aligned}
& p(\text { "larger" } \mid T)= \\
& P\left[\max \left(T, D_{2}, D_{3}, \ldots, D_{n}\right)>-\min \left(T, D_{2}, D_{3}, \ldots, D_{n}\right)\right],
\end{aligned}
$$

which expresses the logic that the correct "larger" response will be given if the largest deviation from zero is in the positive direction, regardless of whether it comes from a target or one of the $n-1$ distractors.

A similar formulation exists for the "smaller" response:

$$
\begin{aligned}
& p\left(\text { "smaller" } \mid T^{\prime}\right)= \\
& P\left[\max \left(T^{\prime}, D_{2}, D_{3}, \ldots, D_{n}\right) \leq-\min \left(T^{\prime}, D_{2}, D_{3}, \ldots, D_{n}\right)\right]
\end{aligned}
$$

The probability correct is given by

$$
P(\text { correct })=P(\text { "larger"। } T) P(T)+P(\text { "smaller"। } T) P\left(T^{\prime}\right)
$$

which is equal to

$$
\begin{aligned}
& P(\text { correct })= \\
& P\left[\max \left(T, D_{2}, \ldots, D_{n}\right)>-\min \left(T, D_{2}, \ldots, D_{n}\right)\right] P(T)+ \\
& P\left[\max \left(T^{\prime}, D_{2}, \ldots, D_{n}\right) \leq-\min \left(T^{\prime}, D_{2}, \ldots, D_{n}\right)\right] P\left(T^{\prime}\right) .
\end{aligned}
$$


This is the probability of a correct response for each type of target times the probability of each target appearing (set by the experimenter to .5 in our case).

To simplify this expression, first consider the case in which the larger target, $T$, is presented:

$P(\operatorname{correct} \mid T)=$

$P\left[\max \left(T, D_{2}, \ldots, D_{n}\right)>-\min \left(T, D_{2}, \ldots, D_{n}\right)\right]$.

To solve this equation, we need to consider a density function equal to the max of the target and $n-1$ distractors $P\left[\max \left(T, D_{2}\right.\right.$, $\left.\left.\ldots, D_{n}\right)=x\right]$ and determine for how many stimuli this quantity dominates that are in the opposite direction $P\left[-\min \left(T, D_{2}, \ldots\right.\right.$, $\left.\left.D_{n}\right)<x\right]$ because if one of these dominates our density function of correct responses, the observer responds incorrectly. This yields the integral

$$
\begin{aligned}
& P(\text { correct } \mid T)=\int_{0}^{\infty} P\left[\max \left(T, D_{2}, \ldots, D_{n}\right)=x\right] \\
& P\left[-\min \left(T, D_{2}, \ldots, D_{n}\right)<x\right] d x,
\end{aligned}
$$

where the first term includes all of the different values of our density function that lead to a correct response, and the second term includes all of the negative values. When the maximum of our positive values exceeds all the negative values, $-\min \left(T, D_{2}, \ldots\right.$, $D_{n}$ ), then one makes the correct response.

Equation A16 is equal to

$$
\begin{array}{r}
\mathrm{P}(\text { correct } \mid \mathrm{T})=\int_{0}^{\infty} P\left[\left(\max \left(D_{2}, \ldots, D_{n}\right)<\mathrm{x}\right)\right. \text { and } \\
\left.\left(-\min \left(D_{2}, \ldots, D_{n}\right)<x\right)\right] P(T=x) d x+ \\
(n-1) \int_{0}^{\infty} P\left[\left(\max \left(T, D_{3}, \ldots, D_{n}\right)<x\right)\right. \text { and } \\
\left.\left(-\min \left(T, D_{3}, \ldots, D_{n}\right)<x\right)\right] P(D=x) d x .
\end{array}
$$

The first term computes the probability that the target wins, and the second term computes the probability that one of the $n-1$ distractors wins. This reduces to

$$
\begin{aligned}
P(\text { "larger" } \mid T)= & \int_{0}^{\infty} f(x-w s)[F(x)-F(-x)]^{(n-1)} d x+ \\
& (n-1) \int_{0}^{\infty} f(x)[F(x-w s)-F(-(x-w s))] \\
& {[F(x)-F(-x)]^{(n-2)} d x . }
\end{aligned}
$$

Assuming Gaussian distributions and set sizes 2 and 8, the predicted $\log -\log$ slope is 0.42 , which is larger than the 0.22 of yes-no and 0.34 of the localization tasks.

\section{Two-Target, N-Alternative Localization}

Computing the probability of choosing the correct target location is chosen is a straightforward modification of the two-target identification equation (Equation A18).

$$
\begin{aligned}
P(\text { correct })= & \int_{0}^{\infty} f(x-w s)[F(x)-F(-x)]^{(n-1)} d x+ \\
& \int_{-\infty}^{0} f(x-w s)[F(-x)-F(x)]^{(n-1)} d x,
\end{aligned}
$$

which computes in the first term the probability that the target item dominates all distractors on its side. The second term computes the probability that the target exceeds all distractors on the other side. Assuming again $P(T)=P\left(T^{\prime}\right)=0.5$ and symmetric distributions, one does not need to include the probabilities for the smaller target $T^{\prime}$. Assuming Gaussian distributions and set sizes of two and eight, the predicted log-log slope is 0.17 for two-target localization.

\section{IFC Identification}

For comparison with the other models, consider the model for a two-interval forced choice (2IFC) task (cf. Palmer et al., 1993). Here $n$ stimuli are presented in two successive intervals and the observer chooses which interval contains the target. With the max rule, the probability correct is

$$
\begin{gathered}
p(c)=p\left[\max \left(T, D_{2}, D_{3}, \ldots D_{n-1}\right)>\max \left(D_{1}, D_{2}, \ldots D_{n}\right)\right] \\
=1-\int_{-\infty}^{\infty} f(x) F(x-w s) F(x)^{2 n-2} d x, \quad
\end{gathered}
$$

Assuming Gaussian distributions and set sizes 2 and 8, the predicted $\log -\log$ slope is 0.31 .

\section{The Overlapping Channels Model}

The overlapping channels model differs from the previous models in that it explicitly breaks the independent processing assumption. Instead, stimuli influence the representations of neighboring stimuli. This lack of independence proves formidable in terms of analytic predictions, and thus we resort to Monte Carlo simulations in order to produce quantitative predictions. Below we describe the procedures used to estimate the mislocalizations found in the $n$-alternative localization experiments. The strategy is to estimate the degree of pooling and then calculate the predicted set-size effect for that degree of pooling.

Following the experiments, consider only set sizes 2 and 8 . The overlapping channels model is fit only to the set-size- 8 data, because the set-size- 2 data have no mislocalizations. That is, in the set-size- 2 condition two positions are chosen to receive stimuli, but the observer can make one of eight responses. However, we find that errors were almost always limited to the position opposite the target location. This is also true for the relevant set-size- 2 condition in Experiment 4 where eight locations received stimuli but only two were pre-cued. Thus the source of mislocalizations does not appear to arise from difficulty translating a location on the screen to a position on the response keypad.

$\mathrm{N}$-alternative localization. To fit the mislocalization data, recall that performance is computed as a function of the difference between targets and distractors. In our experiments, this is the relative angle as plotted in Figure 9. Localization performance varies as a function of the weighting parameter in the overlapping 
channels model. Thus to predict the mislocalization data, we conducted Monte Carlo simulations of 100,000 trials per angle.

Within the Monte Carlo simulation, a single trial with set size 8 is conducted as follows. The values associated with the seven distractor locations are sampled from a Gaussian distribution with mean zero and unit variance. The target distribution is sampled from a distribution with unit variance and mean equal to one of six target-distractor differences being evaluated. The pooling procedure is then applied to the eight numbers using the weighted sum:

$$
s_{i}^{\prime}=\frac{s_{i}+\omega s_{i-1}+\omega s_{i+1}}{1+2 \omega}
$$

where $i$ indexes stimulus location, and $s_{i-1}$ and $s_{i+1}$ are the two stimulus locations immediately adjacent to location $s_{i}$. This representation is circular, such that $s_{i-1}$ equals $s_{n}$ when $i=1$, and $s_{i+1}$ equals $s_{1}$ when $i=n$. The position with the greatest value is then chosen as the response, and the trial is then characterized as either correct, a mislocalization on a nearby item, or an error. Performance as a function of target-distractor difference is fit by a psychometric function, and the threshold stimulus is computed.

Once the threshold stimulus is determined, the full psychometric function is mapped out by selecting six stimulus values at logequal steps. Simulations are run by using each of these values, and these produce a pattern of mislocalizations similar to those found in Figure 9. For comparison with the actual data, the threshold stimulus value is set to be the fourth of the six stimulus values in this series (as was done in the experiments). We simulate onemillion trials per stimulus level, which gives performance predictions that are directly comparable with the data in Figure 9.

This procedure is repeated for different values of $\omega$, the weighting parameter in the pooling process, and we used the value that minimized the root-mean-squared error with the Figure 9 data. For all one-target tasks, we used $\omega=0.38$. For the two-target, $n$-alternative localization task of Experiment 2, we found a larger degree of mislocalizations and used $\omega=0.61$.

Coarse localization. The procedures used to derive predictions for coarse localization data are very similar to those of $n$-alternative localization. The only difference is that the decision rule varies slightly. The location associated with the largest value is chosen after applying the pooling procedure, and this location is used to define the coarse localization response. This produces set-size effects that differ slightly from the $n$-alternative localization set-size effects for a fixed value of the weighting parameter $\omega$, because the effects of pooling only affect stimuli at the boundary.

Yes-no identification. The yes-no identification task does not have mislocalization data, and thus we used the same weighting value as for the $n$-alternative localization task. Given a weighting value, the threshold stimulus can be derived by computing hits and false alarm rates for different values of the target-distractor difference and finding the stimulus associated with hit rates and correct rejection rates of $75 \%$. One complication is that yes-no data involve a decision criterion. We assume that observers place this criterion to balance the hits and correct rejections. In the simulation, hits and correct rejections are computed for a variety of angles and decision criterions in a grid-like fashion, and a plane is fit to the resulting surface for both the hits and correct rejections. By using planar interpolation, the combination of angle and criterion is estimated to produce hits and correct rejections of $75 \%$.
This procedure is repeated twice more with increasingly narrow steps to ensure that our surface approximation procedures do not underestimate performance for the threshold stimulus.

Two-target localization. Predictions for the two-target, $n$-alternative localization are very similar to those of the $n$-alternative localization task. The difference is that the value of the target location must be sampled from one of two distributions in order to provide the two types of targets. We sampled either from a Gaussian distribution with the mean equal to the stimulus value or from a Gaussian distribution with the mean equal to minus the stimulus value. One distribution was chosen randomly with probability .5 prior to sampling. Distractors were sampled from a Gaussian distribution with mean equal to zero.

On each trial, the weighting procedures are the same as described above, and Equation A21 is applied to the values sampled from the various target and distractor distributions. To choose a response, the model picked the location that had the greatest absolute deviation from zero. As with $n$-alternative localization, the threshold stimulus is computed and then a million trials per stimulus condition are used to produce predicted mislocalization and to predict set-size effects for the best-fitting weighting value.

Two-target identification. The two-target identification task does not have mislocalization data, and so the value for the weighting parameter found for the two-target localization data is used. The simulation procedures are similar to those of the twotarget localization task, including the distribution of the target and distractor values and the weighting function applied to these values. The only difference resides in the decision rule. For two-target identification, the Monte Carlo procedures determine whether the maximum deviation from zero is greater than zero or less than zero. If the deviation is greater than zero, the model interprets this as one response, and if it is less than zero, the model interprets this as the other response and computes accuracy accordingly. The threshold stimulus is determined by using the Monte Carlo procedures described above, and this threshold stimulus is used in conjunction with that from set size 2 in order to produce a log-log slope prediction.

\section{Intuitions About Task Differences}

While the signal detection-based models are well defined, more is needed to develop some intuitions about the various predictions. In particular, why does two-target identification have such a large set-size effect compared with that of two-target localization? To address this question, we next develop some intuitions starting from what is known as the two-by-two paradigm.

The two-by-two paradigm was designed to match performance in identification and detection experiments (Graham, 1989; Klein, 1985; Thomas, 1985) and can be adapted to compare identification and localization. It requires two responses, one for each of the two judgments of interest. In a typical application, two displays are presented sequentially. One display has one of two possible targets, and the other display has a blank stimulus. One must make a 2IFC response as to which display contained a target (detection) and another forced choice response as to which of the two targets was present (identification). For a variety of assumptions about the decision rule used in each task, equal performance is predicted for detection and identification. 
Table 2

Comparison of Predicted Set-Size Effects Across Different Tasks

\begin{tabular}{lcc}
\hline & \multicolumn{2}{c}{ Set-size effects } \\
\cline { 2 - 3 } Task & One target & Two targets \\
\hline Two-by-two: 2IFC identification & 0.28 & 0.176 \\
Two-by-two: coarse localization & 0.28 & 0.176 \\
$N$-alternative localization & 0.28 & 0.170 \\
Yes-no identification & 0.18 & 0.14 \\
Two-choice identification & & 0.31 \\
\hline
\end{tabular}

Note. Specific to set sizes 2 and 8 with values normalized for comparison with two-interval forced choice (2IFC; see text).

One can apply this paradigm to identification and localization in accuracy search by presenting two search displays. One display contains a target, and the other display contains only distractors. Observers make two responses: a two-choice identification response indicating which display had the target and a two-choice coarse localization response indicating on which side of the display was the target.

This design makes the same set-size predictions for localization and identification. For the set-sizes-2-versus- 8 comparison, the one-target version (e.g., the target is right leaning and distractors are vertical) has a predicted $\log -\log$ slope of 0.278 , while the two-target version (where the target could be either right or left leaning) has a predicted log-log slope of 0.176 . Thus, all else equal, the two-target case produces a smaller set-size effect for both localization and identification tasks.

The 2IFC paradigm provides a standard against which to compare the other tasks (see Table 2). Consider the $n$-alternative and course localization tasks. The predicted log-log slope is 0.34 for both $n$-alternative and coarse localization tasks, but the 2IFC has two intervals and thus twice as many stimuli. If we double the number of stimuli for the $n$-alternative and coarse localization tasks, both tasks have predicted $\log -\log$ slopes of 0.278 , which matches that of the 2IFC tasks. Thus coarse localization, $n$-alternative localization, and 2IFC predictions match if the number of stimuli is appropriately defined.

Changing the number of potential targets makes the above relation no longer true, which explains in part why the two-target case is different. For the two-target case, the 2IFC predicted set size is 0.176 , and the two-target coarse localization matches that if the set size is doubled. However, the $n$-alternative localization task with two targets and twice the number of stimuli as used in
Experiment 2 (doubled for comparisons with the 2IFC task) does not match. The predicted set-size effect for two-target, $n$-alternative localization is 0.17 , which is close but not the same as the 0.176 for 2IFC and two-target coarse localization.

The predictions diverge much more for the identification tasks. For the one-target case, the 2IFC prediction is 0.28 . For yes-no with double the set size, the prediction is 0.18 . Thus there is a large difference in the set-size effects for 2IFC and yes-no identification.

The two-target identification case also has a difference from 2IFC. The two-target version for 2IFC has a log-log slope prediction of 0.176 . The two-target identification version with the set size doubled (for direct comparison with two-target 2IFC) has a predicted set-size effect of 0.305 , which is quite different. The yes-no, two-target identification task (which we did not study) has a doubled set-size effect of 0.14 . Thus, the difference between yes-no and the two-choice identification produces the major part of the interaction seen in Experiments 1 and 2.

To summarize, our analysis using the 2IFC baseline reveals the following effects:

(a) The two-target task reduces the set-size effect relative to the one-target task, all else being equal. It does not affect the identification-localization comparison per se.

(b) Predicted localization set-size effects are in line with the predictions from the 2IFC task as long as the number of stimuli are doubled to make comparisons with the two-interval task. The two-target, $n$-alternative localization task differs only very slightly from the course localization and 2IFC two-target predictions.

(c) The yes-no and two-target identification tasks both significantly differ from the 2IFC task, and the main reason for these differences arises from the yes-no and 2IFC identification tasks being very different for the two-target case. Thus, the major source of interactions seen in Experiments 1 and 2 arises from the difference between yes-no and two-choice identification in the twotarget case.

The above discussion highlights the need to consider the details of the tasks if meaningful comparisons are to be made. This can be accomplished either through a model-based approach, as we have done, or through close matching of tasks such as with the two-bytwo paradigm (M. Green, 1992). One way or the other, one must address the different decision rules required by each task.

Received January 19, 2007

Revision received September 26, 2007

Accepted October 29, 2007 